Office of Naval Research

Department of the Navy

Contract Nonr 220(35)

SMALL-TIME BEHAVIOR OF UNSTEADY CAVITY FLOWS by

D. P. Wang and T. Yao-tsu Wu

Reproduction in whole or in part is permitted for any purpose of the United States Government

Hydrodynamics Laboratory

Kármán Laboratory of Fluid Mechanics and Jet Propulsion

California Institute of Technology

Pasadena, California

Report No.97-3

March 1963 


\title{
SMALL-TIME BEHAVIOR OF UNSTEADY CAVITY FLOWS
}

\author{
by \\ D. P. Wang and T. Yao-tsu Wu \\ California Institute of Technology
}

A perturbation theory is applied to investigate the small-time behavior of unsteady cavity flows in which the time-dependent part of the flow may be taken as a small-time expansion superimposed on an established steady cavity flow of an ideal fluid. One purpose of this paper is to study the effect of the initial cavity size on the resulting flow due to a given disturbance. Various existing steady cavity-flow models have been employed for this purpose to evaluate the initial reaction of a cavitated body in an unsteady motion. Furthermore, a physical model is proposed here to give a proper representation of the mechanism by which the cavity volume may be changed with time; the initial hydrodynamic force resulting from such change is calculated based on this model.

\section{Introduction}

It has been noted that the free surface flow problems become very difficult to treat exactly when the flow is time-dependent. Some of the principal difficulties involved in this class of problems can be envisaged as follows. First of all, the problem is generally nonlinear because the boundary conditions on the unsteady free surface are nonlinear and because the location of the free boundary is not known á priori. Secondly, unlike the case of steady flows with free boundary, the surface of constant pressure is no longer a surface of constant speed. Consequently, the powerful hodograph method widely used in the steady free boundary problems loses much of its potential and usefulness for possible 
applications in this case. Probably for this reason, only a relatively small number of unsteady free surface problems (of rather special cases) have been treated. Some comprehensive survey and review of the recent literatures are available elsewhere (see Gilbarg[1], Birkoff and Zarantonello[2] and[3]), and hence will not be recited here.

It is worth noting that there is an essential difference between the unsteady flows with and without a free surface. In the determination of the velocity field of an unsteady flow without a free surface (which includes the vortex sheet shed from an oscillating wing), the time appears only as a parameter. The kinematics of the unsteady motion will therefore not differ basically from its corresponding steady flow. On the other hand, for an unsteady flow with a free surface, the time variation affects the flow explicitly through the boundary conditions on the free surface. Therefore, the flow will depend on all its previous time history, which further complicates the detailed analysis and calculation. However, when an established steady cavitating flow (regarded as the basic flow) is given a sudden acceleration, the problem of the small-time behavior of the flow is relatively simple because there will be only a short history of any timevarying disturbance to be considered in this period. It is therefore clear that some difficult problems in unsteady cavity flows can be best handled in the small-time limit since, for one reason, the analysis is relatively straightforward. We hope that such an investigation may cast light on some of the basic features of the unsteady cavity flow, especially the behavior of the large frequency limit in the case of oscillating cavity flows.

The impact problem of the Kirchhoff-Helmholtz flow with a flat plate normal to the stream has been treated by Gurevich [4]. Attempt is made here to generalize the consideration to include the effects of (1) the cavitation number (the cavity size being finite), (2) the angle of attack, and (3) the time variation of the net cavity volume.

The existing mathematical theories for steady cavity flows are seen to be based on some proposed physical models. It is well known that in a positive range of the cavitation number up to moderate values of order unity the agreement between the existing models for steady flows may be 
considered very close[5]. No comparison, however, has ever been made (to the authors' knowledge) between these existing models for the case of unsteady cavity flows. In this paper, the re-entrant jet model, Riabouchinsky's model, and a modified wake model are applied to investigate the small-time behavior of the unsteady cavity flow, including the effects of the cavitation number and the incidence angle of the flow.

The problem related to the possibility for the volume of a finite or infinite cavity to vary with time deserves a particular note. For cavity flows of an incompressible liquid surrounding a vapor cavity, it is obvious that, when the cavity volume changes, the conservation of mass and conservation of volume of the entire flow becomes incompatible because of the difference in the densities of these two phases. Consequently, any variation of the cavity volume must come from a source distribution in the flow with its net strength depending on the time-rate of change of the cavity volume. It has been proposed by one of the authors [6]that as a physical model, the change in the cavity volume can be affected by a source or sink located at the point of infinity. A direct consequence of this source with a time-varying strength in a two-dimensional flow of infinite extent is that it generates a pressure field which is logarithmically singular at infinite distances. To create such a flow it requires an infinite amount of energy. Probably for this reason the treatment of the unsteady partial cavity flow by Timman[7], the work of Geurst[8], of Parkin[9], and of $W u[6]$, have been carried out under the assumption of fixed cavity volume and no source at infinity. In reality, however, the flow is usually finite in extent and never two-dimensional in the large; the pressure singularity at infinity, being outside the flow region, may therefore be regarded as a simplifying idealization, much the same as the representation of a solid body by a distribution of singularities. This physical model for permitting the cavity volume to change has been applied to various cases together with other cavity flow models. It is expected that this variable-cavity-volume model will give a good representation of the flow field near the body-cavity system. The validity of this model of course will have to be verified, directly or indirectly, by future experimental observations. 


\section{General Formulation}

To fix idea, we suppose that for the time $t<0$, a steady plane flow of an incompressible, inviscis fluid past a solid body with a cavity formation has been established, the solution of which is assumed to be given, or can be determined with the aid of some cavity flow models. Suppose now the solid body to which the cavity is attached is given for $t>0$ a sudden accelerated motion; the problem is to evaluate the smalltime behavior of the resultant unsteady cavity flow.

In general, the motion of the rigid boundary may consist of a translation and a rotation. Let $\left(x_{0}, y_{0}\right)$ be a point on the rigid surface $\mathrm{S}_{0}\left(\mathrm{x}_{0}, \mathrm{y}_{0}\right)=0$ in the basic steady flow, and let it be displaced in time $t$ to the position $(x, y)$ with translational velocity $\left(V_{1}(t), V_{2}(t)\right)$ and angular velocity $\omega(t)$. In terms of the complex variable $z=x+i y$ and $\mathrm{V}(\mathrm{t})=\mathrm{V}_{1}+\mathrm{i} \mathrm{V}_{2}$, the motion of $\mathrm{z}$ may be written

$$
d z / d t=V(t)+i \omega(t) z
$$

We shall assume that, for small positive $t, V$ and $\omega$ may be expanded in power series of $t$, starting with the linear term so that

$$
d z / d t=c_{1} t+c_{2} t^{2}+i\left(\omega_{1} t+\omega_{2} t^{2}\right) z+0\left(t^{3}\right)
$$

where $c_{n}=a_{n}+i b_{n}, a_{n}, b_{n}$ and $\omega_{n}$ being real constants. It then follows that for small $t$,

$$
z=z_{0}+\frac{1}{2}\left(c_{1}+i \omega_{1} z_{0}\right) t^{2}+\frac{1}{3}\left(c_{2}+i \omega_{2} z_{0}\right) t^{3}+0\left(t^{4}\right) .
$$

The displaced surface will be denoted by $S(x, y, t)=0$. In fact, we have

$$
S_{0}\left(x_{0}(x, y, t), y_{0}(x, y, t)\right)=S(x, y, t),
$$


regarding (1) as to provide the canonical transformation $x_{0}=x_{0}(x, y, t)$, $y_{0}=y_{0}(x, y, t)$.

From the nature of the body motion it also follows that the complex velocity potential of the flow

$$
f(z, t)=\varphi(x, y, t)+i \psi(x, y, t)
$$

will assume for small $t$ the expansion

$$
f(z, t)=f_{0}(z)+t f_{1}(z)+\frac{1}{2} t^{2} f_{2}(z)+\cdots \cdots
$$

where $f_{n}(z)=\varphi_{n}(x, y)+i \psi_{n}(x, y), n=0,1,2, \cdots$, and $f_{O}(z)$ is the complex velocity potential of the basic flow. The function $\varphi_{1}(x, y)$ may be called the initial acceleration potential. While $\psi_{0}=$ const. gives the streamlines of the basic flow, the harmonic functions $\psi_{n}$ for $n \geqslant l$, being the complex conjugate of $\varphi_{n}$, are introduced solely to make $f_{n}(z)$ analytic functions of $z$. The velocity components are, as usual

$$
u(x, y, t)=\partial \varphi / \partial x, \quad v(x, y, t)=\partial \varphi / \partial y
$$

We shall introduce the complex velocity $w=u-i v$, and $w_{n}=d f_{n} / d z$, then from (6)

$$
w(z, t)=w_{0}(z)+t w_{1}(z)+\frac{1}{2} t^{2} w_{2}(z)+\cdots
$$

Similarly the pressure $p(x, y, t)$ may be assumed to possess the expansion

$$
p(x, y, t)=p_{0}(x, y)+p_{1}(x, y)+t p_{2}(x, y)+t^{2} p_{3}(x, y)+\cdots \cdot
$$

where $p_{0}$ denotes the pressure field of the basic flow, $p_{1}$ the impulsive pressure due to the sudden acceleration. Then from the Bernoulli equation,

$$
p / p+\partial \varphi / \partial t+\frac{1}{2}(\nabla \varphi)^{2}=p_{\infty} / \rho+\frac{1}{2} U^{2}
$$


where $p_{\infty}$ and $U$ are the pressure and speed of the basic flow at infinity, we obtain, by equating the coefficients of same powers of $t$, the following relations:

$$
\begin{gathered}
p_{0} / \rho+\frac{1}{2}\left(\nabla \varphi_{0}\right)^{2}=p_{\infty} / \rho+\frac{1}{2} U^{2} \\
P_{1} / \rho=-\varphi_{1}, \\
p_{2} / \rho=-\varphi_{2}-\left(\nabla \varphi_{0}\right) \cdot\left(\nabla \varphi_{1}\right), \text { and so on. }
\end{gathered}
$$

The boundary conditions of the problem are as follows:

(i) At the solid surface, the normal component of the flow velocity relative to the moving boundary must vanish. An alternative way of stating this condition is that the fluid particles originally on $S(x, y, t)=0$, at small time interval apart, will remain on it. That is

$$
\frac{\mathrm{DS}}{\mathrm{Dt}}=\frac{\partial S}{\partial \mathrm{t}}+\frac{\partial \varphi}{\partial \mathrm{x}} \frac{\partial S}{\partial \mathrm{x}}+\frac{\partial \varphi}{\partial \mathrm{y}} \frac{\partial S}{\partial \mathrm{y}}=0 \quad \text { on } \mathrm{S}(\mathrm{x}, \mathrm{y}, \mathrm{t})=0
$$

which becomes, upon using (4),

$$
\frac{\partial \mathrm{S}_{\mathrm{O}}}{\partial \mathrm{x}_{\mathrm{O}}}\left[\frac{\partial \varphi}{\partial \mathrm{x}} \frac{\partial \mathrm{x}_{\mathrm{O}}}{\partial \mathrm{x}}+\frac{\partial \varphi}{\partial \mathrm{y}} \frac{\partial \mathrm{x}_{\mathrm{O}}}{\partial \mathrm{y}}+\frac{\partial \mathrm{x}_{\mathrm{o}}}{\partial \mathrm{t}}\right]+\frac{\partial \mathrm{S}_{\mathrm{O}}}{\partial \mathrm{y}_{\mathrm{O}}}\left[\frac{\partial \varphi}{\partial \mathrm{x}} \frac{\partial \mathrm{y}_{\mathrm{O}}}{\partial \mathrm{x}}+\frac{\partial \varphi}{\partial \mathrm{y}} \frac{\partial \mathrm{y}_{\mathrm{o}}}{\partial \mathrm{y}}+\frac{\partial \mathrm{y}_{\mathrm{o}}}{\partial \mathrm{t}}\right]=0
$$

on $S(x, y, t)=0$. Here the functions $x_{0}(x, y, t)$ and $y_{0}(x, y, t)$ can be written down immediately from (3) by interchanging $z$ and $z_{0}$, and by changing the signs of $a_{n}, b_{n}$ and $\omega_{n}$, as can readily be seen from (3). Equation (11) is in a form convenient for manipulation since $\partial S_{0} / \partial x_{0}$ and $\partial S_{0} / \partial y_{0}$ already correspond to the components of the normal to the initial surface $S_{0}\left(x_{0}, y_{0}\right)=0$. Substituting (3) and (6) in (11), expanding the various quantities about $\left(\mathrm{x}_{\mathrm{o}}, \mathrm{y}_{\mathrm{o}}\right)$ and $\mathrm{t}=0$, and equating the coefficients of different powers of $t$, we obtain the conditions that on $S_{0}\left(x_{0}, y_{0}\right)=0$,

$$
\partial \varphi_{0} / \partial \mathrm{n}_{0}=0
$$




$$
\partial \varphi_{1} / \partial \mathrm{n}_{0}=\mathrm{n}_{0}\left(\mathrm{a}-\mathrm{w}_{1} \mathrm{y}_{0}\right)+\mathrm{n}_{0}\left(\mathrm{~b}_{1}+\omega_{1} \mathrm{x}_{0}\right)
$$

$$
\begin{aligned}
\frac{\partial \varphi_{2}}{\partial n_{0}}=2 n_{0}\left(a_{2}-\omega_{2} y_{0}\right)+2 n_{0}\left(b_{2}+\omega_{2} x_{0}\right)-\omega_{1}\left[n_{0} \frac{\partial \varphi_{0}}{\partial y_{0}}-n_{0} \frac{\partial \varphi_{0}}{\partial x_{0}}\right] \\
-\left(a_{1}-\omega_{1} y_{0}\right)\left[n_{0} \frac{\partial^{2} \varphi_{0}}{\partial x_{0}^{2}}+n_{0} \frac{\partial^{2} \varphi_{0}}{\partial x_{0} \partial y_{0}}\right]-\left(b_{1}+\omega_{1} x_{0}\right)\left[n_{0} \frac{\partial^{2} \varphi_{0}}{\partial x_{0} \partial y_{0}}+n_{0} \frac{\partial^{2} \varphi_{0}}{\partial y_{0}^{2}}\right]
\end{aligned}
$$

where $\vec{n}_{0}=\left(n_{0}, n_{0}\right)$ is the unit outward normal to the surface $S_{0}\left(x_{0}, y_{0}\right)=0$.

(ii) There are two boundary conditions at the free surface of the cavity. Suppose the free surface may be expressed as

$$
F(x, y, t)=y-h(x, t)=0
$$

then the kinematic condition that the fluid particles on the free surface will remain on it requires

$$
\mathrm{v}=\partial \mathrm{h} / \partial \mathrm{t}+\mathrm{u} \partial \mathrm{h} / \partial \mathrm{x} \quad \text { on } \mathrm{y}=\mathrm{h}(\mathrm{x}, \mathrm{t})
$$

We assume that for small $t, h(x, t)$ may be expanded as

$$
h(x, t)=h_{0}(x)+t h_{1}(x)+\frac{1}{2} t^{2} h_{2}(x)+0\left(t^{3}\right)
$$

where $y=h_{0}(x)$ denotes the cavity boundary of the basic flow on which

$$
d h_{0} / d x=v_{o}\left(x, h_{0}\right) / u_{0}\left(x, h_{0}\right)
$$

Substituting (7) and (14) in (13), and expanding $u_{n}$ and $v_{n}$ on $y=h(x, t)$ about $y=h_{0}(x)$ and $t=0$, we obtain

$$
\begin{aligned}
& h_{1}=v_{0}\left(x, h_{0}\right)-u_{0}\left(x, h_{0}\right) d h_{0} / d x=0, \\
& h_{2}=v_{1}\left(x, h_{0}\right)-u_{1}\left(x, h_{0}\right) d h_{0} / d x .
\end{aligned}
$$


Since $h_{1} \equiv 0$, the free surface will not be displaced in the time of order $t$, as should be expected.

In the basic flow, the cavity boundary is a surface of constant pressure, and hence also one of constant velocity, say

$$
\mathrm{p}_{\mathrm{o}}=\mathrm{p}_{\mathrm{c}}, \quad\left|\nabla \varphi_{\mathrm{o}}\right|=\mathrm{q}_{\mathrm{C}}, \quad \text { on } \mathrm{y}=\mathrm{h}_{\mathrm{o}}(\mathrm{x})
$$

so that by the Bernoulli equation (10),

$$
q_{c}=U(1+\sigma)^{\frac{1}{2}}
$$

where $\sigma$ is the cavitation number of the basic flow, defined as

$$
\sigma=\left(p_{\infty}-p_{c}\right) / \frac{1}{2} \rho U^{2}
$$

We shall assume that the pressure in the cavity of the unsteady flow will be maintained at the same constant value $\mathrm{p}_{\mathrm{c}}$, that is,

$$
p(x, y, t)=p_{c} \quad \text { on } y=h(x, t)
$$

By expanding the left side of this dynamic condition about $y=h_{0}(x)$ and $t=0$, using (8), (14) and (16), the following conditions result

$$
\mathrm{p}_{1}=\mathrm{p}_{2}=0 \quad \text { on } \mathrm{y}=\mathrm{h}_{\mathrm{O}}(\mathrm{x})
$$

Hence from (10),

$$
\begin{array}{ll}
\varphi_{1}=0 & \text { on } \mathrm{y}=\mathrm{h}_{\mathrm{O}}(\mathrm{x}), \\
\varphi_{2}=-\left(\nabla \varphi_{0}\right) \cdot\left(\nabla \varphi_{1}\right) & \text { on } \mathrm{y}=\mathrm{h}_{\mathrm{O}}(\mathrm{x}) .
\end{array}
$$

Upon differentiating (2la) along $y=h_{0}(x)$ and using (15), it is readily seen that $\left(\nabla \varphi_{0}\right) \cdot\left(\nabla \varphi_{1}\right)=0$, and hence

$$
\varphi_{2}=0 \quad \text { on } \mathrm{y}=\mathrm{h}_{\mathrm{o}}(\mathrm{x}) .
$$

From ( $21 a)$ and ( $21 b)$ it therefore follows that

$$
\left(\nabla \varphi_{0}\right) \cdot\left(\nabla \varphi_{1}\right)=\left(\nabla \varphi_{0}\right) \cdot\left(\nabla \varphi_{2}\right)=0 \quad \text { on } y=h_{0}(x)
$$


which asserts that the perturbation velocity is normal to the original cavity boundary up to the time of order $t^{2}$.

(iii) At the point of infinity we require the perturbation velocity to vanish,

$$
\left|\nabla \varphi_{n}\right| \rightarrow 0 \text { as } \quad|z| \rightarrow \infty, \quad \text { for } \quad n=1,2, \cdots
$$

If, in addition to the sudden acceleration of the solid body and the assumption of the constancy of the cavity pressure, a certain amount of fluid is removed at infinity with a source strength $Q_{1} t+\frac{1}{2} Q_{2} t^{2}+\cdots$ so that the cavity volume may be changed arbitrarily, then, aside from condition (23), we must impose additional conditions at infinity as

$$
\operatorname{Im} \int_{\Gamma} w_{n}(z) d z=Q_{n}, \quad n=1,2, \cdots
$$

where $\Gamma$ is a contour around the point of infinity. However, we impose no boundedness condition on $p$ at infinity since, if such $Q_{n}$ can be arbitrarily chosen, $\mathrm{p}_{\mathrm{n}}(\mathrm{n} \geqslant 1)$ will be logarithmically singular at infinity. It will be seen later that, when the cavity is taken to be infinitely long (Helmholtz flow, corresponding to the cavitation number $\sigma=0$ ), the solution exists only when $Q_{n}=0$. Consequently the effect of change in cavity volume can be sought only in the case of finite cavities. However, the limit of the hydrodynamic forces in such cases as the cavity becomes infinitely long, with $Q_{n}$ held fixed, is seen to exist.

On the other hand, from Kelvin's theorem on the conservation of circulation, the circulation around the point of infinity cannot be changed in the unsteady motion for $t<\infty$. By combining this condition with (24), we may write

$$
\int_{\Gamma} w_{n}(z) d z=i Q_{n}, \quad n=1,2,3 \cdots
$$

This completes our formulation of the small-time perturbation theory. 


\section{Solution of the Perturbed Flow.}

For the moment we assume that the solution of the basic steady flow is given. We note that in the basic flow the entire boundary of the body-cavity system belongs to a streamline, the form of which is known. Therefore, by the general theory of conformal mapping, it is always possible to find an analytic function

$$
\zeta(z)=\xi(x, y)+i \eta(x, y)
$$

such that the entire flow region in the $z$-plane is mapped by (26) into the upper half $\zeta$-plane with the entire boundary of the body-cavity system mapped onto the entire $\xi$-axis. For simplicity, we shall make the part $|\xi|<1$ of the real $\zeta$-axis correspond to the wetted solid surface and the part $|\xi|>1$ to the cavity boundary.

After the transformation to the $\zeta$-plane the boundary value problem formulated in the last section can be stated as a Hilbert problem, as will be shown below, the solution of which is readily attained. It is noted from the last section that the problems of different orders in the perturbation are expressed in a similar form. That is, the normal velocities $\partial_{\varphi_{n}} / \partial n_{0}$ are given at the initial solid surface and the potentials $\varphi_{n}$ are prescribed at the unperturbed cavity boundary. It is therefore sufficient to treat one as the typical case. To save writing we shall denote $\mathrm{f}_{\mathrm{n}}=\varphi_{\mathrm{n}}+\mathrm{i} \psi_{\mathrm{n}}$ by $F=\Phi+i \Psi$.

It is convenient to introduce the analytic function

$$
\mathrm{G}(\zeta)=\mathrm{dF} / \mathrm{d} \zeta=(\mathrm{dF} / \mathrm{d} z)(\mathrm{d} z / \mathrm{d} \zeta)
$$

which is defined for $\operatorname{Im} \zeta>0$. Then on the solid surface, $\eta=0+,|\xi|<1$,

$$
\operatorname{Im} G=-\frac{\partial \Phi}{\partial \eta}=-\frac{\partial \Phi}{\partial n_{0}}\left|\frac{d z}{d \zeta}\right|=g_{1}(\xi) \quad \text { say }
$$

which is known, by (12) and (26). Furthermore, on the cavity boundary, $\eta=0+,|\xi|>1$, 


$$
R \ell G=\partial \Phi / \partial \xi=g_{2}(\xi) \quad \text { say }
$$

which is also known, by (21). In particular, $g_{2}=0$ for $\varphi_{1}$ and $\varphi_{2}$. Finally, (25) becomes

$$
\int_{\Gamma} \frac{d F}{d z} d z=\int_{\Gamma^{\prime}} G(\zeta) d \zeta=i Q
$$

where $\Gamma^{\prime}$ is the image of $\Gamma$ in the Gplane, the subscript $n$ of $Q$ is dropped to show a typical case.

It is possible to transform the above boundary value problems of a mixed type into a Hilbert problem by extending the unknown function $G(\zeta)$ to a sectionally analytic function, defined on the whole $\zeta$-plane (excluding the real $\zeta$-axis if necessary). Since $\varphi_{3}$ and higher terms will not betreated explicitly here, we shall demonstrate the method by taking $g_{2}$ of (29) to be zero; the general case of $g_{2} \neq 0$ can be carried out similarly. First, the function $G(\zeta)$ may be continued into the lower half $\zeta$-plane by

$$
G(\bar{\zeta})=-\overline{G(\zeta)}
$$

For the case $\mathrm{g}_{2}=0, \mathrm{G}(\bar{\zeta})$ is the analytical continuation of $G(\zeta)$ through the interval $|\xi|>1$. In the following, $G^{ \pm}(\xi)$ will be used to denote the limiting value of $G(\zeta)$ as $\eta \longrightarrow \pm 0$. From (28), (29) and (31) it then follows that

$$
\begin{array}{ll}
G^{+}+G^{-}=2 i \operatorname{Im} G^{+}=2 i g_{1}(\xi) & \text { for }|\xi|<1, \\
G^{+}-G^{-}=2 \mathrm{Rl} G^{+}=0 & \text { for }|\xi|>1 .
\end{array}
$$

The above Hilbert problem is well-known [10], its general solution can be written

$$
G(\zeta)=\frac{i}{\pi}\left(\zeta^{2}-1\right)^{-\frac{1}{2}} \int_{-1}^{1} \frac{\left(1-\xi^{2}\right)^{\frac{1}{2}} g_{1}(\xi)}{\xi-\zeta} \mathrm{d} \xi+\frac{i P(\zeta)}{\left(\zeta^{2}-1\right)^{\frac{1}{2}}} \text { for } \eta>0
$$


where the function $\left(\zeta^{2}-1\right)^{\frac{1}{2}}$ is defined on the entire $\zeta$-plane with the branch so chosen that $\left(\zeta^{2}-1\right)^{\frac{1}{2}} \rightarrow \zeta$ as $|\zeta| \rightarrow \infty$, and $P(\zeta)$ is an arbitrary Laurent's series with real coefficients. The last term in (33) is the general solution to the homogeneous problem (with $g_{1}=0$ also). The real coefficients of $P(\zeta)$, and hence $G(\zeta)$, can be determined uniquely, when (30) is satisfied and the condition that the pressure is integrable over the rigid boundary is observed.

In the following the above perturbation theory will be carried out for several basic steady cavity flows.

\section{Inclined Lamina in Kirchhoff Flow}

As a simple example we consider the basic flow to be that past a flat plate held at an angle $\alpha$, with a cavity formation of infinite length. The solution of this problem is known [11], which we simply cite below for the subsequent use. The coordinate system in the $z$-plane and its conformal mapping planes are shown in figure l. For simplicity the free stream velocity $U$ and the plate length $\ell$, are normalized to unity. The solution $\mathrm{w}_{\mathrm{O}}=\mathrm{w}_{\mathrm{O}}\left(\mathrm{z}_{\mathrm{O}}\right)$ can be written parametrically as

$$
\begin{aligned}
\mathrm{w}_{0} & =\left(\sqrt{1-\zeta^{2}}-1\right) / \zeta, \\
\mathrm{z}_{\mathrm{o}} & =-\mathrm{K} \int_{1}^{\zeta}\left(\sqrt{1-\zeta^{2}}+1\right)(\zeta+\sec \alpha)^{-3} \mathrm{~d} \zeta \\
& =\frac{K}{2}\left\{\frac{1+\sqrt{1-\zeta^{2}}}{(\zeta+\sec \alpha)^{2}}-\frac{\cot \alpha}{\sin \alpha} \frac{\sqrt{1-\zeta^{2}}}{(\zeta+\sec \alpha)}+2 \cot ^{3} \alpha \tan ^{-1}\left(\sqrt{\frac{1-\zeta}{1+\zeta}} \tan \frac{\alpha}{2}\right)-(1+\sec \alpha)\right.
\end{aligned}
$$

where

$$
K=2 \tan ^{3} \alpha \sin \alpha /(4+\pi \sin \alpha) .
$$

The entire flow region is mapped by (34) into the upper half $\zeta$-plane with the corresponding boundary as prescribed in the last section (i.e. on $\eta=0,|\xi|<1$ corresponds to the plate and $|\xi|>1$ to the 
cavity surface). The coefficient of the normal force $N_{0}$ of the basic flow is

$$
C_{N_{0}}=N_{0} /\left(\frac{1}{2} \rho U^{2} \ell\right)=2 \pi \sin \alpha /(4+\pi \sin \alpha)
$$

(a) The first order solution

The unit normal to the plate is now $\vec{n}_{0}=(0,-1)$. We suppose the rotational motion is referred to the leading edge of the plate. Then the boundary conditions (12b), (2la) and (23) become

$$
\begin{array}{lr}
\partial \varphi_{1} / \partial y_{0}=\left(b_{1}+\omega_{1} x_{0}\right) & \text { on } \eta=0,|\xi|<1,(36 \mathrm{a}) \\
\varphi_{1}=0 \text { and hence } \partial \varphi_{1} / \partial \xi=0 & \text { on } \eta=0,|\xi|>1 .(36 b) \\
\left|\nabla \varphi_{1}\right| \longrightarrow 0 & \text { as } \zeta \longrightarrow-\sec \alpha .
\end{array}
$$

It is noted that $a_{1}$, the first term of the $x$-component of acceleration, drops out from (36a), implying that the acceleration of the plate parallel to itself has no effect on the flow up to time of order t. Furthermore,(25) cannot be satisfied unless all the $Q_{n}=0$. This can be seen as follows. If $Q_{n} \neq 0$, then (25) implies that $w_{n}=Q_{n} / 2 \pi z+o\left(|z|^{-1}\right)$, and hence $\varphi_{\mathrm{n}} \sim\left(\mathrm{Q}_{\mathrm{n}} / 2 \pi\right) \log |\mathrm{z}|$, as $|\mathrm{z}| \rightarrow \infty$. It follows that $\mathrm{p}_{\mathrm{n}}$ will be logarithmically singular at $\mathrm{z}=\infty$, which contradicts the conditions of $\mathrm{p}_{\mathrm{n}}$, such as (20), on the undisturbed free boundary $y=h_{0}(x)$ which extends to infinity. This indicates that the Kirchhoff-Helmholtz model with an infinite cavity is not a realistic model for the consideration of change of cavity volume. The problem when $Q_{n} \neq 0$ will be considered later when other finite-cavity models are adopted.

By making use of (36a), (36b) and (34b), (28) and (29) become

$$
\begin{array}{rlrl}
\operatorname{Im} G=\frac{\partial \varphi_{1}}{\partial y_{0}}\left|\frac{d z}{d \xi}\right| & =K\left(b_{1}+\omega_{1} x_{0}\right)\left(\sqrt{1-\xi^{2}}+1\right)(\xi+\sec \alpha)^{-3}, & |\xi|<1, \\
\operatorname{R\ell } G=0, & |\xi|>1,
\end{array}
$$


where $x_{0}(\xi)$ is given by $(34 b)$, and $K$ is given by $(34 c)$. Finally, by (33), we obtain

$$
\frac{d f}{d \zeta}=\frac{K}{\pi\left(1-\zeta^{2}\right)^{\frac{1}{2}}}\left\{\int_{-1}^{1} \frac{\left(b_{1}+\omega_{1} x_{0}\right)\left(1-\xi^{2}+\sqrt{1-\xi^{2}}\right)}{(\xi-\zeta)(\xi+\sec \alpha)^{3}} d \xi+\sum_{n=-\infty}^{\infty} C_{n}(\zeta+\sec \alpha)^{n}\right\}
$$

where $C_{n}$ are real coefficients. The Laurentz series in the last term is expanded about $\zeta=-\sec \alpha(\operatorname{or} z=\infty)$ for the convenience of application of the boundary conditions at $z=\infty$. The first term in (37) behaves like $\zeta^{-2}$ as $|\zeta| \rightarrow \infty$ and is regular everywhere except at $\zeta= \pm 1$. Now by using $(34 \mathrm{~b})$,

$$
w_{1}=\frac{d f}{d \zeta} \frac{d \zeta}{d z}=-\frac{(\zeta+\sec \alpha)^{3}}{K\left(\sqrt{1-\zeta^{2}}+1\right)} \frac{d f_{1}}{d \zeta} .
$$

From (37) and (38) we readily see that $C_{n}=0$ except for $n=-1,-2$ in order that $w_{1} \longrightarrow 0$ as $z \longrightarrow \infty$ (or $\left.\zeta \longrightarrow-\sec \alpha\right)$ and $w_{1}$ is regular as $\zeta \overleftrightarrow{\mathbb{I}} \infty$. Furthermore, with $Q_{n}=0$, as explained above, (30) requires that

$$
\mathrm{zw}_{1} \rightarrow 0 \text { as }|\mathrm{z}| \longrightarrow \infty
$$

From (34b), we find that as $|z| \longrightarrow \infty$,

$$
z \sim \frac{K}{2} \frac{1+i \tan \alpha}{(\zeta+\sec \alpha)^{2}} .
$$

Making use of this result, we readily deduce from (37) and (38) that (39) is satisfied if, and only if, $\mathrm{C}_{-1}=\mathrm{C}_{-2}=0$ also. Therefore all the coefficients $C_{n}$ vanish, thereby the first order solution given by (37) and (38) is uniquely determined.

It may be noted that $w_{1}$ has a singularity at the edges of the plate, physically corresponding to a narrow spray sheet. For, with $\xi= \pm(1+\epsilon),|\epsilon|<<1$, we deduce from (37) and (38) that as $\xi \longrightarrow \pm 1$, 


$$
w_{1} \sim \frac{i}{\pi} \frac{( \pm 1+\sec \alpha)^{3}}{(2 \epsilon)^{\frac{1}{2}}} \int_{-1}^{1}\left(\frac{1+\xi}{1-\xi}\right)^{ \pm \frac{1}{2}}\left[\frac{\left(b+\omega x_{0}\right)\left(1+\sqrt{1-\xi^{2}}\right)}{(\xi+\sec \alpha)^{3}}\right] d \xi\left[1+0\left(|\epsilon|^{\frac{1}{2}}\right)\right]
$$

From the behavior of $w_{1}$ on the free surface ( $\epsilon$ positive small) it may be seen that the free surface starts to move into the cavity when the plate accelerates into the fluid $\left(e \cdot g_{0}\right.$, with $\left.b_{1}<0, \omega_{\mathbb{1}}=0\right)$, and vice versa. Furthermore, it is noted that $w_{1}$ is of order $|z|^{-3 / 2}$ for large values of $|z|$. The net effect can be seen to be such that there will be no net change in the cavity volume.

Since the spray sheets do not produce any singular force (unlike the leading edge suction on a thin airfoil), the normal force acting on the plate can be obtained by integrating the pressure along the plate so that for small $t$, in view of (3),

$$
\begin{aligned}
N & =\int_{0}^{1} p\left(x_{0}, 0-, t\right) d x_{0}=\int_{0}^{1}\left[p_{0}\left(x_{0}, 0\right)+p_{1}\left(x_{0}, 0\right)+t p_{2}\left(x_{0}, 0\right)\right] d x_{0}+0\left(t^{2}\right) . \\
& =N_{0}+N_{1}+t N_{2}+0\left(t^{2}\right) .
\end{aligned}
$$

The first term $N_{0}$ is given by (35). Now, from (10)

$$
N_{1}=\int_{0}^{1} p_{1}\left(x_{0}, 0\right) d x_{0}=-\rho \int_{0}^{1} \varphi_{1}\left(x_{0}, 0\right) d x_{0}=-\rho \int_{-1}^{1} x_{0}(\xi) \frac{\partial \varphi(\xi, 0)}{\partial \xi} d \xi .
$$

By substituting the real part of (37) for $\partial \varphi_{1} / \partial \xi$ in the above integral, the normal force coefficient may be expressed as

$$
\mathrm{C}_{N_{1}}=N_{1} /\left(\frac{1}{2} \rho U^{2} \ell\right)=-\left(\frac{l b}{U^{2}}\right) \Gamma_{b_{1}}-\left(\frac{L^{2} \omega}{U^{2}}\right) \Gamma_{\omega_{1}},
$$

where

$$
\begin{aligned}
& \Gamma_{\mathrm{b}}(\alpha)=-\frac{2}{\pi} \int_{-1}^{1} \frac{\mathrm{x}_{\mathrm{o}}(\xi) \mathrm{d} \xi}{\sqrt{1-\xi^{2}}} \int_{-1}^{1} \frac{\sqrt{1-s^{2}}}{\mathrm{~s}-\xi} \frac{\partial \mathrm{x}_{\mathrm{o}}(\mathrm{s})}{\partial \mathrm{s}} \mathrm{ds} \\
& \Gamma_{\omega}(\alpha)=-\frac{1}{\pi} \int_{-1}^{1} \frac{\mathrm{x}_{\mathrm{o}}(\xi) \mathrm{d} \xi}{\sqrt{1-\xi^{2}}} \int_{-1}^{1} \frac{\sqrt{1-s^{2}}}{\frac{\partial \mathrm{x}_{0}^{2}(s)}{\partial s}} \mathrm{ds}
\end{aligned}
$$


the above integrals being interpreted by their Cauchy principal values. In (4la), $b_{1}$ and $\omega_{1}$ are expressed in the physical units, and the plate length $\ell$ and free stream velocity $U$ are restored for completeness. The integrals in (4lb) and (4lc) cannot be expressed in terms of elementary functions of $\alpha$; they are integrated numerically and the results are plotted versus $\alpha$ in figure 5. In particular, we have

$$
\Gamma_{\mathrm{b}_{1}}(\pi / 2)=0.8448
$$

which is the special case treated by Gurevich [4]. The quantity $\mathrm{C}_{N_{1}}$ represents the jump in the normal force coefficient due to the acceleration.

(b) The second order solution for $\alpha=\pi / 2, \omega_{1}=b_{2}=\omega_{2}=0$.

To facilitate investigation of the behavior of the second order solution, let us choose the special case: $\alpha=\pi / 2$ (the flat plate being held normal to the stream) and $\omega_{1}, b_{2}, \omega_{2}$ all vanish. Then the boundary conditions (12c), (21), and (23) become

$$
\begin{aligned}
& \partial \varphi_{2} / \partial \mathrm{y}_{\mathrm{o}}=-\mathrm{b}_{1} \partial^{2} \varphi / \partial \mathrm{y}_{\mathrm{o}}^{2}, \quad \text { on } \quad \eta=0, \quad|\xi|<1 \text {, } \\
& \varphi_{2}=0 \quad \text { on } \eta=0, \quad|\xi|>1, \\
& \left|\nabla \varphi_{2}\right| \longrightarrow 0 \quad \text { as }|z| \longrightarrow \infty
\end{aligned}
$$

Since the component $a_{1}$ of the acceleration parallel to the plate does not appear in the above conditions, the flow will not be affected by it up to the second order terms. Now, in the limit as $\alpha \longrightarrow \pi / 2$, the zeroth order solution becomes

$$
z_{0}=\left[2(1-\zeta)-\zeta\left(1-\xi^{2}\right)^{\frac{1}{2}}+\cos ^{-1} \zeta\right] /(4+\pi)
$$

and $w_{0}$ is still given by (34a). Consequently, from (28) and (29) 


$$
\begin{array}{ll}
\operatorname{Im} G=b_{1} \frac{\partial u_{0}}{\partial x}\left|\frac{\partial x}{\partial \xi}\right|=b_{1} \xi^{-2}\left[\left(1-\xi^{2}\right)^{-\frac{1}{2}}-1\right], & \eta=0,|\xi|<1, \\
\operatorname{R\ell G}=0 & \eta=0,|\xi|>1 .
\end{array}
$$

Hence, by (33),

$$
\frac{d f_{2}}{d \xi}=\frac{b_{1}}{\pi \sqrt{1-\zeta^{2}}} \int_{-1}^{1} \frac{1-\left(1-\xi^{2}\right)^{\frac{1}{2}}}{(\xi-\zeta) \xi^{2}} d \xi
$$

in which the complementary solution is zero, as can be shown by the same argument given previously for $\varphi_{1}$. Carrying out the integration, we find

$$
\begin{aligned}
& \frac{\mathrm{df}}{\mathrm{d} \zeta}=\frac{\mathrm{b}_{1}}{\pi \zeta^{2}}\left(1-\zeta^{2}\right)^{-\frac{1}{2}}\left\{2 \zeta+\log \frac{\zeta-1}{\zeta+1}-i \pi\left(1-\zeta^{2}\right)^{\frac{1}{2}}\right\} \\
& \mathrm{w}_{2}=\frac{\mathrm{df}}{\mathrm{dz}}=-\frac{\mathrm{i}(4+\pi)}{4 \zeta^{2}}\left[1-\left(1-\zeta^{2}\right)^{\frac{1}{2}}\right] \frac{\mathrm{df}}{\mathrm{d} \zeta} .
\end{aligned}
$$

It is readily verified that $w_{2}$ also behaves like $z^{-3 / 2}$ for large $|z|$.

The second order normal force, by (40a) and (10), can be obtained from

$$
\begin{aligned}
N_{2} & =\int_{0}^{1} p_{2}\left(x_{0}, 0\right) d x_{0}=\rho \int_{-1}^{1}\left[\varphi_{2}+u_{0} u_{1}\right]_{\eta=0} \frac{\partial x_{0}}{\partial \xi} d \xi \\
& =\rho \int_{-1}^{1}\left[-x_{0}(\xi) \frac{\partial \varphi_{2}}{\partial \xi}+u_{0}(\xi) u_{1}(\xi) \frac{\partial x_{0}}{\partial \xi}\right] d \xi .
\end{aligned}
$$

After substituting the various terms and evaluating the resulting integral, we find the simple result:

$$
\mathrm{N}_{2}=0
$$

For this special case we therefore have the normal force coefficient 


$$
\begin{aligned}
C_{N}(t) & =C_{N_{0}}+C_{N_{1}}+C_{N_{2}} t+0\left[(U t / \ell)^{2}\right] \\
& =0.8798+0.8448\left(\ell b_{1} / U^{2}\right)+0(U t / \ell)^{2} .
\end{aligned}
$$

Thus for constant acceleration, $C_{N}$ has, aside from the stepwise change, the following behavior

$$
\left(d C_{N} / d t\right)_{t=0+}=0
$$

However, $\mathrm{N}_{2}$ may not vanish when $\mathrm{b}_{2}, \omega_{1}$, and $\omega_{2}$ are different from zero.

The quantity $m_{1}=N_{1} / b_{1}$ may be called the initial induced mass of this cavity flow, then by (48)

$$
m_{1}=\frac{1}{2} \rho U^{2} \ell C_{N_{1}} b_{1}^{-1}=0.4224 \rho \ell^{2} .
$$

If the flat plate had undergone an acceleration $b_{1}$ normal to the flow without wake formation (a postulated Dirichlet flow), then the induced mass would be

$$
m_{0}=\pi \rho(\ell / 2)^{2}
$$

Thus

$$
\mathrm{m}_{1} / \mathrm{m}_{0}=0.5377
$$

This ratio is less than unity, as should be expected, since the cavitated side of the plate, being exposed to constant pressure, has no capacity of imparting kinetic energy to the exterior flow.

\section{Re-entrant Jet Model}

We proceed to consider the effect of the cavitation number o (defined by (18)) on the unsteady flow when the cavity of the basic flow is finite in size. An additional degreee of freedom achieved in this group of flow problems is that the cavity volume can now be changed arbitrary by prescribing a flow source at infinity. In order that the basic steady flow 
is tractable to analysis, resort may be made to various cavity-flow models known in literature, such as the Riabouchinsky model [12], the re-entrant jet model [1], the wake model proposed independently by Joukowsky [13], Roshko [14] and Eppler [15], and the modified wake model introduced by Wu [16]. In each of these flow models an artifice of some sort is introduced to admit $\sigma$ as a free parameter, and to replace the actual wake flow of a real fluid by a simplified model within the framework of an equivalent potential flow. It has been found that in a positive range of $\sigma$ up to moderate values of order unity, the agreement between these models may be considered very close (see Wu [5]). Furthermore, the validity of these models in predicting the hydrodynamic forces acting on the body has been supported by experimental observations. The purpose of the following several sections is to make a comparison between the resultant unsteady flows when different models are used for the basic cavity flow, for such a task should be of fundamental value in the study of unsteady cavity flows.

For simplicity the basic flow is taken to be that past a flat plate held normal to the stream of unperturbed velocity $U$ and pressure $P_{\infty}$, forming a finite cavity with a prescribed cavity pressure $\mathrm{p}_{\mathrm{c}}\left(<\mathrm{p}_{\infty}\right)$. According to the re-entrant jet model, the free streamlines eventually reverse their direction at the rear part of the cavity, forming a reentrant jet which disappears on another "Riemann Sheet" (see figure 2). Due to the assumed symmetry of the flow, it suffices to consider only the flow in the left half physical z-plane. To save writing, both the half plate length, $\ell / 2$, and the constant speed $q_{C}$ (see (18)) on the cavity surface will be normalized to unity.

It is convenient to introduce the variable

$$
\Omega=\log \frac{\mathrm{dz}}{\mathrm{df}_{\mathrm{O}}}=\log \frac{1}{\mathrm{w}_{\mathrm{O}}}=\log \frac{1}{\mathrm{q}_{\mathrm{O}}}+\mathrm{i} \theta
$$

where $f_{o}$ is the complex potential of the basic flow, $q_{0}$ is the flow speed, and $\theta$ the flow inclination with respect to the $x$-axis. The part of the flow under consideration in the $z, f_{O}$, and $\Omega$-planes is shown in figure 2 . By applying the Schwarz-Christoffel transformation, the flow region can be 
mapped conformally into the upper half $\zeta$-plane, with the point of infinity I, front stagnation E, plate edge $A$, jet infinity $B$ and the rear stagnation $C$ corresponding to $\zeta=\infty, 0,1, b$, and $c$ respectively. The required transformation is given by

$$
\begin{gathered}
\frac{\mathrm{df}_{\mathrm{O}}}{\mathrm{d} \zeta}=\mathrm{A} \frac{\zeta-\mathrm{c}}{\zeta-b}, \\
\frac{\mathrm{d} \Omega}{\mathrm{d} \zeta}=\frac{\mathrm{B}}{\zeta(\zeta-c)[(\zeta-1)(\zeta-b)]^{\frac{1}{2}}}
\end{gathered}
$$

where A, B are two coefficients. From the local behavior of $\Omega$ at $\zeta=0$ and $c$, we find the relations

$$
B=-\frac{1}{2} c b^{\frac{1}{2}}, \quad b=c(c-1)\left(c-\frac{3}{4}\right)^{-1} \text {. }
$$

Integrating (53), and by making use of (54), we obtain

$$
\frac{d z}{d f_{0}}=e^{\Omega}=\frac{[(c-b)(\zeta-1) /((c-1)(\zeta-b)\}]^{\frac{1}{2}}+1}{[(c-b)(\zeta-1) /((c-1)(\zeta-b))]^{\frac{1}{2}}-1}\left\{\frac{[(\zeta-b) /\{b(\zeta-1)\}]^{\frac{1}{2}}+1}{\left[(\zeta-b) /\{b(\zeta-1)]^{\frac{1}{2}}-1\right.}\right\}^{\frac{1}{2}} .
$$

As $|\zeta| \rightarrow \infty, \mathrm{df}_{0} / \mathrm{dz} \rightarrow \mathrm{U}=(1+\sigma)^{-\frac{1}{2}}$. From this condition and (55) it results

$$
(2+\sigma) / \sigma=\frac{1}{2}(c-1)^{\frac{1}{2}} c^{3 / 2}\left(c-\frac{3}{4}\right)^{-3 / 2} .
$$

Finally, by integrating (55) and (52) to obtain $z=z(\zeta)$, the coefficient $A$ is determined by the plate length, giving

$$
A=(b-1)^{3 / 2} /[K(1)-K(0)] \text {, }
$$

where 


$$
\begin{aligned}
K(\xi) & =(2 c-b)[b \xi(1-\xi)]^{\frac{1}{2}}+(2 c-1)[\xi(b-\xi)]^{\frac{1}{2}}+\left(c-\frac{1}{2} b\right) \sin ^{-1} \frac{2 \xi-b}{b} \\
& +b^{\frac{1}{2}}\left[b\left(b-\frac{3}{2}\right)+c(2-b)\right] \sin ^{-1}(2 \xi-1)+(b-1)^{3 / 2}(c-b) \sin ^{-1} \frac{(2 b-1) \xi-b}{b-\xi}
\end{aligned}
$$

This completes the zeroth order solution for prescribed $\sigma$. In particular, on the half plate, $\mathrm{y}=0,-1<\mathrm{x}<0$ (or $\eta=0+, 0<\xi<1$ ), we have

$$
\begin{gathered}
x(\xi)=-[K(\xi)-K(0)] /[K(1)-K(0)] \\
\frac{d x}{d \xi}=A\left(\frac{c-\xi}{b-\xi}\right) \frac{[(c-b)(1-\xi) /\{(c-1)(b-\xi)\}]^{\frac{1}{2}}+1}{\left[(c-b)(1-\xi) /\{(c-1)(b-\xi)]^{\frac{1}{2}}-1\right.}\left\{\frac{[(b-\xi) /[b(1-\xi)\}]^{\frac{1}{2}}+1}{[(b-\xi) /[b(1-\xi)\}]^{\frac{1}{2}}-1}\right\}^{\frac{1}{2}}
\end{gathered}
$$

This expression is needed for the first order solution.

When a sudden acceleration of magnitude $b_{1}$ is applied normal to the plate, the boundary conditions corresponding to (32) can be written

$$
\begin{aligned}
& \mathrm{G}^{+}+\mathrm{G}^{-}=-2 \mathrm{i} \mathrm{b}_{1} \mathrm{dx} / \mathrm{d} \xi \quad \text { for } \quad 0<\xi<1 \\
& G^{+}-G^{-}=0 \quad \text { for } \quad 1<\xi<b \text {, } \\
& \mathrm{G}^{+}+\mathrm{G}^{-}=0 \quad \text { for } \quad-\infty<\xi<0 \text { and } \mathrm{b}<\xi<\infty \text {. }
\end{aligned}
$$

The last condition (59c) expresses the assumption that the perturbed flow preserves the basic flow symmetry about the y-axis, that is, $v_{1}=0$ on $\eta=0$, for $\xi<0$ and $\xi>b$. The above conditions are expressedin the form different from (32). The solution, however can be written down directly by applying the same principle. It is obvious that $H(\zeta)=[(\zeta-1)(\zeta-b)]^{\frac{1}{2}}$, defined with branch cuts from $\zeta=-\infty$ to 1 and from b to $\infty$ so that $H \rightarrow \zeta$ as $|\zeta| \rightarrow \infty, 0<\arg \zeta<\pi$, is a homogeneous solution of the present problem. Therefore the general solution can be written 


$$
G(\zeta)=\frac{b_{1}}{\pi H(\zeta)} \int_{0}^{1} \frac{[(1-\xi)(b-\xi)]^{\frac{1}{2}}}{\xi-\zeta} \frac{d x}{d \xi} d \xi+\frac{1}{H(\zeta)}\left[\sum_{-\infty}^{\infty} c_{n} \xi^{n}\right]
$$

where $d x / d \xi$ is given by $(58 b)$ and $c_{n}$ are arbitrary real constants. To determine ${ }^{n}$, we note first that the pressure must be finite at $\zeta=0$, hence $c_{n}=0$ for $n<0$. Furthermore, we note that the first term in (60) is of order of $\xi^{-2}$ as $|\zeta| \rightarrow \infty$, which implies that the behavior of $\mathrm{df}_{1} / \mathrm{dz}$ at large distances is determined by the last term in (60). By applying condition (30) and using the symmetry property of the flow, we obtain $c_{0}=Q_{1} / 2 \pi, c_{1}=c_{2}=\ldots .=0$, where $Q_{1}$ is the source strength defined in (24).

The integral in (60) can be integrated explicitly. For the determination of hydrodynamic forces, however, only the real part of $G$ on the plate (where $\eta=0,0<\xi<1$ ) is needed. The final result is

$$
\begin{aligned}
R \ell G=- & \frac{b_{1}}{\pi} \frac{[(1-\xi)(b-\xi)]^{-\frac{1}{2}}}{[K(1)-K(0)]}\left\{A_{1}+B_{1}(\xi-1)\right. \\
& \left.+\sqrt{b} \frac{(\xi-1)[(2 c-b-2)(\xi-1)-c(b-1)]}{[\xi(b-\xi)]^{\frac{1}{2}}} \log \frac{(b-1) \xi+(b-\xi)+2 \sqrt{(b-1) \xi(b-\xi)}}{b(1-\xi)}\right\} \\
& -\frac{Q_{1}}{2 \pi}[(1-\xi)(b-\xi)]^{-\frac{1}{2}}
\end{aligned}
$$

where

$$
\begin{aligned}
& A_{1}=\pi\left[b(c-1)+\frac{1}{2}\right]+\frac{\sqrt{b}}{2}\left(b^{2}-2 b+2 c\right)\left(\frac{\pi}{2}+\sin ^{-1} \frac{2-b}{b}\right)-\sqrt{b(b-1)}(b-2 c), \\
& B_{1}=\sqrt{b}(b-2 c)\left(\frac{\pi}{2}+\sin ^{-1} \frac{2-b}{b}\right)-\pi(2 c-1) .
\end{aligned}
$$

By using (40b) and the symmetry property of the flow, the first order normal force $\mathrm{N}_{1}$ is given by

$$
N_{1}=-2 \rho \int_{0}^{1} x(\xi)\{R \ell G\} d \xi
$$

where $x(\xi)$ and $R \ell G$ are given by (58a) and (61). The result can be expressed in terms of nondimensional parameters as 


$$
C_{N_{1}}=N_{1} /\left(\frac{1}{2} \rho U^{2} \ell\right)=-\left(\frac{l b}{U^{2}}\right) \Gamma_{b_{1}}-\left(\frac{Q_{1}}{U^{2}}\right) \Gamma_{Q_{1}},
$$

in which the coefficients $\Gamma_{b_{1}}$ and $\Gamma_{Q_{1}}$ are functions of the cavitation number $\sigma$. Analytic evaluation of the above integral is too tedious to be practical. The numerical computation of these coefficients has been carried out with an IBM 7090, the final result is plotted versus $\sigma$ in figures 6 and 7 to show the effect of (i) the finite cavity size and (ii) the change in the cavity volume. From this result several salient features of significance may be pointed out.

First, it is noted that as $\sigma \rightarrow 0$, the value of $\Gamma_{\mathrm{b}}$ tends to 0.8448, which is the limit of Kirchhoff-Helmholtz case (see equation (42)). From small to moderately large values of $\sigma, \Gamma_{b_{1}}$ increases very slowly with increasing $\sigma$ as compared with the rate of increase of the zeroth order drag, which increases approximately with the factor $(1+\sigma)$ (see, e.g., Gilbarg [1]).

Another point of interest is that $\Gamma_{Q_{1}} \rightarrow 0$ rather rapidly as $\sigma \longrightarrow 0$, this limit being independent of $Q_{1}$ so long as $Q_{1}$ is finite. This result shows that the effect on the drag force of removing fluid at infinity is insignificant when the cavity is sufficiently long. Furthermore, it shows that the limit of the solution as $\sigma \longrightarrow 0$ is non-uniform with respect to $Q_{1}$ since the solution in the Kirchhoff case does not exist unless $Q_{1}=0$.

\section{Riabouchinsky's Model}

The essential feature of the Riabouchinsky model is the introduction of an appropriate image body downstream of the real body so that the free boundaries of the cavity are connected by this pair of solidboundaries. Let us apply this model to consider the cavity flow past a flat plate set normal to the stream, the flow in the physical $\mathrm{z}$-plane is shown in figure 3. Again, as in the problem stated in the previous section, the unperturbed velocity and pressure are $U$ and $p_{\infty}$ respectively, the 
cavity pressure is $\mathrm{P}_{\mathrm{C}}$, corresponding to the cavitation number $\sigma$. Also the half plate length, $\ell / 2$, and the constant speed $\mathrm{q}_{\mathrm{C}}$ on the cavity will be normalized to unity. Furthermore, due to the assumed symmetry, only the left half $z$-plane of the flow need be considered.

For the present case it is convenient to denote by $\zeta$ the complex velocity potential. We further introduce an auxiliary complex variable $\tau$ defined by

$$
\tau=\frac{1}{2}\left(w_{0}+w_{0}^{-1}\right)
$$

where $\mathrm{w}_{\mathrm{O}}=\mathrm{d} \zeta / \mathrm{dz}$ is the hodograph plane of the basic flow. The flow field under consideration in the $z$-plane and conformal mapping planes $w_{0}, \zeta, \tau$, are shown in figure 3. By the assumed symmetry of the flow, we may choose the potential at the front and rear stagnation points to be $\zeta=-n$ and $n$ respectively, and $\zeta_{B}=-m, \zeta_{D}=m$. We further define $\mathrm{k}^{\prime}$ and $\mathrm{k}$ by

$$
\mathrm{k}^{\prime}=\frac{1}{1+\sigma}, \quad \mathrm{k}=\left(1-\mathrm{k}^{\prime 2}\right)^{\frac{1}{2}}=\left(2 \sigma+\sigma^{2}\right)^{\frac{1}{2}} /(1+\sigma) .
$$

Then, at $z=\infty$, we have

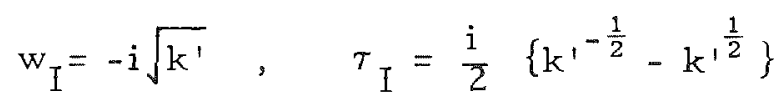

The upper half $\tau$-plane is mapped into the upper half $\zeta$-plane by the Schwarz-Christoffel transformation

$$
\zeta=2 n \sqrt{k^{\prime}} \tau\left[4 k^{\prime} \tau^{2}+\left(1-k^{\prime}\right)^{2}\right]^{-\frac{1}{2}} .
$$

From the local conformal behavior of $\zeta(\tau)$ at the point $D$, i.e. $\zeta_{\mathrm{D}}=\mathrm{m}, \tau_{\mathrm{D}}=1$, we find the relationship

$$
m=2 n \sqrt{k^{\prime}} /\left(1+k^{\prime}\right) .
$$

Let us introduce another auxiliary variable $\omega=\mu+i \nu$ defined by

$$
w_{0}=\frac{d \zeta}{d z}=-i \frac{\sqrt{k^{\prime}}}{\mathrm{dn} \omega} \text {, }
$$

where dn $w$ is the Jacobian elliptic function, delta amplitude of $\omega$, with modulus $k$. In the following analysis the conventional notations (see [17]) for the elliptic functions and integrals will be used without specification and the modulus $\mathrm{k}$ will always be omitted to save writing. By substituting (68) and (63) into (66) it gives 


$$
\zeta=i \frac{\mathrm{n}}{\mathrm{k}} \frac{\mathrm{dn}^{2} \omega-\mathrm{k}^{\prime}}{\sin \omega \operatorname{cn} \omega}
$$

From (68) and (69) we deduce that on both the front and the image half plates $-1<\mathrm{x}<0$,

$$
x=\frac{n}{\sqrt{k^{\prime}}}\left[k^{\prime} \mu-E(\mu)+k^{\prime}\left(1-k^{\prime}\right) \frac{\operatorname{sn} \mu \operatorname{cn} \mu}{\operatorname{dn} \mu}\right] \quad, \quad 0<\mu<\frac{K}{2} .
$$

Evaluating the above result at $\mu=\mathrm{K} / 2$, which corresponds to $\mathrm{x}=-1$, we obtain

$$
n=\frac{2 \sqrt{k^{\prime}}}{E-k^{\prime} K+\frac{\left(1-k^{\prime}\right)^{2}}{1+k^{\prime}}} \text {, }
$$

which completes the necessary calculation for the basic flow.

Due to the presence of the image plate, an additional assumption is needed for this model in the study of its small time behavior. It is given that at $t=0$ a sudden acceleration $b_{1}$ is applied on the front plate directed in the positive y-direction. For small $t>0$ the image plate may be assumed to move in the y-direction with speed

$$
v=-\beta b_{1} t+0\left(t^{2}\right)
$$

where $\beta$ is an unknown constant. To determine this unknown constant $\beta$, we shall assume that on the image plate the jump in the drag due to the suddenly applied acceleration is zero. The physical significance of this assumption may be explained as follows. It has been pointed out by Wu [5] that the image plate in the basic flow may be regarded as a means to represent the energy dissipation in the wake flow of a real fluid. In fact, in a frame of reference at rest with respect to the fluid at infinity, the work done by the moving image plate is negative and numerically equal to the work done by the real plate since the total force on the pair of plates vanishes. This negative work done by the image plate therefore corresponds to the mechanical energy removed from the 
system in unit time as there is no other means of dissipating energy in potential flow. Now in the unsteady motion, it is conceivable that the rate of dissipation in the wake (of the actual flow) cannot be affected at small time. This implies that the initial change of momentum at the image plate must vanish. It is this physical meaning that underlies the above assumption. For small $t>0$, the boundary condition on the part of the $\xi$-axis $\left(\eta=0^{+}\right)$corresponding to the image plate can be written by using (72) as

$$
\operatorname{Im} G=\beta b_{1} R \ell\left(\frac{d z}{d \xi}\right)
$$

where $G=d f / d \zeta$. Or, by applying the continuation (31), we have on the part of the $\xi$-axis corresponding to the image plate the condition

$$
\mathrm{G}^{+}+\mathrm{G}^{-}=2 \text { i } \beta b_{1} \mathrm{Rl}\left(\frac{\mathrm{dz}}{\mathrm{d \zeta}}\right) \text {. }
$$

Referring to conditions (32) and (73) and using (68), the boundary conditions of this problem are

$$
\begin{array}{ll}
G^{+}+G^{-}=-2 i b_{1} R ?\left(\frac{d z}{d \zeta}\right)=2 i \frac{1}{\sqrt{k^{\prime}}} \frac{c n \mu(\xi)}{\operatorname{sn} \mu(\xi)} & \text { for }-n<\xi<-m \\
G^{+}-G^{-}=0 & \text { for }-m<\xi<m \\
G^{+}+G^{-}=2 i \beta b_{1} R \ell\left(\frac{d z}{d \zeta}\right)= & 2 i \frac{1}{\sqrt{k^{1}}} \frac{c n \mu(\xi)}{\operatorname{sn} \mu(\xi)} \text { for } m<\xi<n \\
G^{+}+G^{-}=0 & \text { for }-\infty<\xi<-n, n<\xi<\infty ;
\end{array}
$$

where $m$ and $n$ are given by (67) and (71).

Consider the function $h(\zeta)=\sqrt{\zeta^{2}-m^{2}}$ with the branch cuts from $-\alpha$ to $-m$ and from $m$ to $\infty$, and $h(\xi) \longrightarrow \zeta$ as $|\zeta| \rightarrow \infty$, $0<\arg \zeta<\pi$. This $h(\zeta)$ is obviously a solution of the corresponding homogeneous Hilbert problem and satisfies (31). Therefore, by using (33), the required solution may be written

$$
\begin{aligned}
& G(\zeta)=-\frac{b_{k} k^{-\frac{1}{2}}}{\pi \sqrt{\xi^{2}-m^{2}}}\left\{\int_{-n}^{-m \sqrt{\xi^{2}-m^{2}} \frac{c n \mu(\xi)}{s n \mu(\xi)}} \frac{n-\beta}{\xi-\zeta} d \xi-\frac{\sqrt{\xi^{2}-m^{2} \frac{c n \mu(\xi)}{s n \mu(\xi)}}}{\xi-\zeta} d \xi\right\} \\
& +\frac{P(\zeta)}{\sqrt{\zeta^{2}-m^{2}}}
\end{aligned}
$$


where $P(\zeta)=\sum_{-\infty}^{\infty} c_{n} \xi^{n}$, and $c_{n}$ are arbitrary real constants. Again, since the pressure at $\zeta=0$ should be finite and the first term in (74) is of order of $\xi^{-2}$ as $|\zeta| \rightarrow \infty$, we have, by applying condition (30) and the symmetric property of the flow, $P(\zeta)=Q_{1} / 2 \pi$. By using (69), the variable $\xi$ in (74) can be transformed into the variable $\mu$, giving

$$
\begin{aligned}
G(\zeta)= & \frac{b_{1}\left(1-k^{\prime}\right) n}{\pi \sqrt{k^{\prime}} \sqrt{\zeta^{2}-m^{2}}}\left\{\int_{0}^{K / 2} \frac{\operatorname{cn}^{2} \mu d n^{2} \mu\left(1-\frac{k^{\prime}}{d^{2} \mu}\right)^{2}}{\operatorname{dn}^{2} \mu+\frac{1+k^{\prime}}{n} \zeta \operatorname{dn} \mu+k^{\prime}} d \mu\right. \\
& \left.+\beta \int_{0}^{K / 2} \frac{\operatorname{cn}^{2} \mu \operatorname{dn}^{2} \mu\left(1-\frac{k^{\prime}}{\mathrm{dn}^{2} \mu}\right)^{2}}{\operatorname{dn}^{2} \mu-\frac{1+k^{\prime}}{n} \zeta \operatorname{dn}^{2}+k^{\prime}} d \mu\right\} \\
& +\frac{Q 1}{2 \pi \sqrt{\zeta^{2}-m^{2}}} \cdot
\end{aligned}
$$

After integrating the above expression and by using (69) we deduce that

$$
\begin{aligned}
& \mathrm{R} \ell \mathrm{G}=\mp \frac{\mathrm{b}}{\mathrm{l}}\left\{( 1 + \beta ) \left[\mathrm{A}_{2}\left(\frac{1+\mathrm{k}^{\prime}}{\mathrm{n}}\right)\left(\frac{\mathrm{dn} \mu}{\mathrm{dn}^{2} \mu-\mathrm{k}^{\prime}}\right)+\frac{\mathrm{K}}{2 \sqrt{\mathrm{k}^{\prime}}}\left(\frac{\mathrm{dn}^{2} \mu-\mathrm{k}^{\prime}}{\mathrm{dn} \mu}\right)\right.\right. \\
& +\frac{1}{\sqrt{\mathrm{k}^{1}}} \frac{\mathrm{dn} \mu \mathrm{cn}^{2} \mu}{\operatorname{sn}^{2} \mu} \pi^{*}\left(\sin ^{-1} \frac{1}{\sqrt{1+\mathrm{k}^{1}}}, \frac{1}{\operatorname{sn}^{2} \mu}\right)-\mathrm{k}^{, 5 / 2} \frac{\operatorname{sn}^{2} \mu}{\operatorname{dn} \mu \mathrm{cn}^{2} \mu} \text {. } \\
& \left.=\pi\left(\sin ^{-1} \frac{1}{\sqrt{1+k^{\prime}}}, \frac{\mathrm{dn}^{2} \mu}{\operatorname{cn}^{2} \mu}\right)\right] \pm(1-\beta)\left[B \frac{\mathrm{dn}^{2} \mu+k^{\prime}}{\mathrm{dn}^{2} \mu-\mathrm{k}^{\prime}}+\frac{1}{2} \frac{\left(\mathrm{dn}^{2} \mu-\mathrm{k}^{\prime}\right) \mathrm{k}^{-\frac{1}{2}}}{\left(1-\mathrm{k}^{\prime}\right) \sin \mu \mathrm{cn} \mu}\right. \text {. } \\
& \left.\left.\cdot \log \frac{\operatorname{cn} \mu+\sqrt{k^{\prime}} \operatorname{sn} \mu}{\operatorname{cn} \mu-\sqrt{k^{\prime} \sin \mu}}\right]\right\} \mp \frac{Q}{2 \pi} \frac{1+k^{\prime}}{n}\left(\frac{\operatorname{dn} \mu}{\operatorname{dn}^{2} \mu-k^{\prime}}\right) \quad 0<\mu<\frac{K}{2}
\end{aligned}
$$

where the upper sign is used for the front plate, the lower sign for the image plate, $\pi^{*}$ denotes the function which corresponds to the Cauchy principal value of the integral representation of the function $\pi$ (for the definition, see [17]), and 


$$
\begin{gathered}
A_{2}=\frac{1-k^{\prime}}{1+k^{\prime}} \frac{E+k^{\prime} K+\left(1+k^{\prime}\right)}{E-k^{\prime} K+\frac{\left(1-k^{\prime}\right)^{2}}{\left(1+k^{\prime}\right)}} \\
B_{2}=\frac{1+k^{\prime}}{2 \sqrt{k^{\prime}}}\left[\frac{\pi}{2} \frac{1-k^{\prime}}{1+k^{\prime}}+\sin ^{-1} \frac{1-k^{\prime}}{1+k^{\prime}}\right] .
\end{gathered}
$$

By applying (40b), (70), (76a) and (66), we obtain the normal force $\mathrm{N}_{1}$ on the front plate as

$$
N_{1}=2 \rho\left\{b\left[(1+\beta) I_{1}+(1-\beta) I_{2}\right]+\Omega_{1} I_{3}\right\}
$$

and the normal force $\mathrm{D}_{\mathrm{y}}$ (in the positive $\mathrm{y}$-direction) on the image plate as

$$
D_{y}=2 \rho\left\{b_{1}\left[(1+\beta) I_{1}-(1-\beta) I_{2}\right]+Q_{1} I_{3}\right\}
$$

where

$$
\begin{aligned}
& I_{1}=\frac{\left(1-k^{\prime}\right) n}{\pi \sqrt{k^{\prime}}} \int_{0}^{K / 2} x(\mu) \frac{\operatorname{sn} \mu \mathrm{cn} \mu}{\operatorname{dn} \mu}\left\{\frac{\left(1+k^{\prime}\right) \sqrt{k^{\prime}}}{n} A_{2}+\frac{K}{2} \frac{\left(\operatorname{dn}^{2} \mu-k^{\prime}\right)^{2}}{\operatorname{dn}^{2} \mu}\right. \\
& +\left(\mathrm{dn}^{2} \mu-\mathrm{k}^{\prime}\right)\left[\frac{\mathrm{cn}^{2} \mu}{\operatorname{sn}^{2} \mu} \pi^{*}\left(\sin ^{-1} \frac{1}{\sqrt{1+k^{1}}}, \frac{1}{\operatorname{sn}^{2} \mu}\right)\right. \\
& \left.\left.-\mathrm{k}^{3} \frac{\operatorname{sn}^{2} \mu}{\operatorname{dn}^{2} \mu \mathrm{cn}^{2} \mu} \pi\left(\sin ^{-1} \frac{1}{\sqrt{1+k^{\prime}}}, \frac{\operatorname{dn}^{2} \mu}{\mathrm{cn}^{2} \mu}\right)\right)\right\} \mathrm{d} \mu \\
& I_{2}=\frac{\left(1-k^{\prime}\right) n}{\pi \sqrt{k^{\prime}}} \int_{0}^{K / 2} x(\mu)\left[\sqrt{k^{\prime}} B\left(\frac{\operatorname{dn}^{2} \mu+k^{\prime}}{\operatorname{dn}^{2} \mu}\right) \operatorname{sn} \mu c n \mu+\frac{1}{2} \frac{\left(\operatorname{dn}^{2} \mu-k^{\prime}\right)^{2}}{\left(1-k^{\prime}\right) \mathrm{dn}^{2} \mu} .\right. \\
& \left.\cdot \log \frac{\operatorname{cn} \mu+\sqrt{k^{\prime}} \operatorname{sn} \mu}{\operatorname{cn} \mu-\sqrt{k^{\prime}} \operatorname{sn} \mu}\right] d \mu \\
& I_{3}=\frac{1-k^{\prime 2}}{2 \pi} \int_{0}^{K / 2} x(\mu) \frac{\operatorname{sn} \mu \operatorname{cn} \mu}{\operatorname{dn} \mu} d \mu
\end{aligned}
$$


and $x(\mu)$ is given by $(70)$.

Now we apply the condition that on the image plate the change in the drag due to the applied acceleration is zero, giving

$$
2 \rho b_{1}\left[(1+\beta) I_{1}-(1-\beta) I_{2}\right]=0 \text {. }
$$

or

$$
\beta=\frac{I_{2}-I_{1}}{I_{1}+I_{2}}
$$

Substituting (80) into (77), we obtain the drag on the front plate as

$$
N_{1}=4 \rho\left[b_{1} \frac{2 I_{1} I_{2}}{I_{1}+I_{2}}+\frac{Q_{1}}{2} I_{3}\right] .
$$

This result can also be expressed in the following non-dimensional form:

$$
C_{N_{1}}=N_{1} /\left(\frac{1}{2} \rho U^{2} \ell\right)=-\left(\frac{l b}{U^{2}}\right) \Gamma_{b_{1}}-\left(\frac{Q_{1}}{U^{2}}\right) \Gamma_{Q_{1}}
$$

where

$$
\Gamma_{b_{1}}=-4 \frac{I_{1} I_{2}}{I_{1}+I_{2}} \quad \Gamma_{Q_{1}}=-2 I_{3}
$$

The integrals $I_{1}, I_{2}$ and $I_{3}$ are computed numerically with an IBM 7090 and the final result of $\Gamma_{b_{1}}$ and $\Gamma_{Q_{1}}$ are shown in figures 6 and 7 as a comparison with the results of other flow models. It is further noted that as $\sigma \rightarrow 0, \Gamma_{b_{1}}$ reduces to $(42)$ of the Kirchhoff case and $\Gamma_{Q_{1}} \rightarrow 0$.

\section{A Wake Model for an Oblique Plate with a Finite Cavity}

Thus far we have considered the accelerating motion of an inclined plate in Kirchhoff flow, and of the finite-cavity flow past a plate broadwise to the stream. Application of either the re-entrant jet model or the Riabouchinsky model to an oblique plate with a finite cavity formation leads to very complicated analyses. The task is considerably simplified, however, if we adopt a modified wake model recently proposed by Wu[16] 
to describe the basic steady flow. The purpose of this section is to determine the effect of acceleration on the hydrodynamic forces with the cavitation number $\sigma$ and the angle of attack $\alpha$ as two free parameters.

The basic flow is taken to be a uniform stream of infinite extent impinging on a flat plate at an incidence angle $\alpha$, to which a finite cavity is attached. According to this modified wake model, the incoming stagnation streamline branches off the plate at the leading edge $A$ and the trailing edge $B$, forming two free streamlines ACI and BC'I which are assumed to become asymptotically parallel to the main flow at downstream infinity (see figure 4). The pressure on the parts AC and BC' of the free streamlines is assumed to take the constant cavity pressure $\mathrm{P}_{C}$, and the space within the closed curve ACC'BA is regarded to represent approximately the cavity. The space in between the free streamlines CI and C'I represents a crude model of the dissipating wake, along its boundary the uniform stream conditions are eventually restored at downstream infinity. The flow outside this infinite wake strip is assumed to be irrotational. The locations of the points $C$ and $C^{\prime}$ are determined with two assumptions; the first is that both the velocity potential and the flow inclination at $C$ and $C^{\prime}$ are equal, and the second assumption is the so-called "hodograph-slit condition" that the free streamlines CI and C'I form a slit of undetermined shape in the hodograph plane. With these two additional assumptions the whole flow field outside the wake is then completely determined. For the convenience of subsequent application, the solution of the basic flow is reproduced briefly in the following. The plate length $\ell$ and the constant speed $\mathrm{q}_{\mathrm{C}}$ on $\mathrm{AC}$ and $\mathrm{BC}^{\prime}$ are again normalized to unity.

The flow in the physical $z$-plane, the complex potential $f_{0}$-plane, and the hodograph $w_{0}-$ plane are shown in figure 4. The subscript of $w_{0}$ will be omitted for simplicity. We further introduce the parametric $\zeta$-plane defined by

$$
\zeta=\frac{1}{2}\left(w+w^{-1}\right)
$$


or

$$
w=\zeta-\left(\zeta^{2}-1\right)^{\frac{1}{2}}
$$

in which the function $\left(\zeta^{2}-1\right)^{\frac{1}{2}}$ is defined with a branch cut made between the points $\zeta=-1$ and 1 so that $\left(\zeta^{2}-1\right)^{\frac{1}{2}} \rightarrow \zeta$ as $|\zeta| \rightarrow \infty$. At the point of infinity, the complex velocity takes the value

$$
\mathrm{W}=\mathrm{W}=\mathrm{U} \mathrm{e}^{-\mathrm{i} \alpha}, \quad \mathrm{U}=(1+\sigma)^{-\frac{1}{2}} .
$$

The corresponding value of $\zeta$ is

$$
\zeta_{\infty}=\frac{1}{2}\left(U^{-1} e^{i \alpha}+U e^{-i \alpha}\right)
$$

Since $\operatorname{Im} \mathrm{f}_{\mathrm{O}}=0$ on the entire real $\zeta$-axis, the complex potential $\mathrm{f}_{0}(\zeta)$ can be continued analytically into the lower-half $\zeta$-plane by

$$
f_{0}(\bar{\zeta})=\overline{f_{0}(\zeta)}
$$

Now from the asymptotic behavior of the streamlines $\psi_{0}=$ const. near the point $\zeta=\zeta_{\infty}$, it is evident that $f_{0}$ must have there a simple pole. Furthermore, from the local conformal behavior of $f_{0}$ at $f_{0}=0$, it is obvious that $f_{0}=O\left(\zeta^{-2}\right)$ as $|\zeta| \rightarrow \infty$. Therefore the solution must be of the form

$$
f_{0}=\frac{A}{4} \frac{1}{\left(\zeta-\zeta_{\alpha}\right)\left(\zeta-\bar{\zeta}_{\infty}\right)}
$$

where $A$ is a real constant. Or, expressing in terms of $w$ by (83),

$$
f_{0}=\frac{A w^{2}}{(w-W)(w-W)\left(w-W^{-1}\right)\left(w-W^{-1}\right)} \text {. }
$$

The $z$-plane is determined by integration of $d z / d f=1 / w$, giving

$$
\begin{aligned}
z+a= & \frac{f_{o}(w)}{w}+i B\left\{\left(\frac{1}{\bar{W}}-\bar{W}\right)\left[\frac{1}{W} \log (w-W)-W \log \left(w-\frac{1}{W}\right)\right]\right. \\
& \left.-\left(\frac{1}{W}-W\right)\left\{\frac{1}{\bar{W}} \log (w-\bar{W})-\bar{W} \log \left(w-\frac{1}{\bar{W}}\right)\right]\right\},(88 a)
\end{aligned}
$$


where the constant $B$ is related to $A$ by

$$
\overline{\mathrm{B}}=2\left(\frac{1}{\mathrm{U}}-\mathrm{U}\right) \sin \alpha\left[\left(\frac{1}{\mathrm{U}}+\mathrm{U}\right)^{2}-(2 \cos \alpha)^{2}\right] .
$$

Finally, the constant $A$ is determined by the plate length as

$$
\begin{gathered}
A=\left[\left(\frac{1}{U}+U\right)^{2}-(2 \cos \alpha)^{2}\right] / K \\
K=2 \frac{\left(U^{-1}+U\right)^{2}+(2 \cos \alpha)^{2}}{\left(U^{-1}+U\right)^{2}-(2 \cos \alpha)^{2}}+\frac{\pi\left(U^{-1}+U\right)}{2 \sin \alpha}+\frac{\left(U^{-1}+U\right)^{2}-(2 \cos \alpha)^{2}}{\left(U^{-1}-U\right) \sin \alpha} \tan ^{-1}\left(\frac{U^{-1}-U}{2 \sin \alpha}\right) .
\end{gathered}
$$

For the unsteady motion we shall confine ourselves to the simple case of constant acceleration so that for any point $z$ of the plate,

$$
\mathrm{d} z / d t=\left(a_{1}+i b_{1}\right) t
$$

Then the boundary conditions of this problem (see equation 32) become

$$
\begin{array}{ll}
G^{+}+G^{-}=-2 i b_{1} R l(d z / d \zeta) & \text { for }|\xi|>1, \\
G^{+}-G^{-}=0 & \text { for }|\xi|<1,
\end{array}
$$

where $\mathrm{G}=\mathrm{df} / \mathrm{d} \zeta$. In the first condition, $\mathrm{dz} / \mathrm{d} \zeta=\mathrm{w}^{-1} \mathrm{df} / \mathrm{d} \zeta$ can be deduced from (83) and (87).

Similar to the general solution of the problem with the boundary conditions given in (32), the general solution of the above boundary value problem, stated in (91), can be written

$$
G(\xi)=\frac{b_{1}}{\pi\left(\zeta^{2}-1\right)^{\frac{1}{2}}}\left\{\int_{-\infty}^{-1}-\int_{1}^{\infty}\right\} \frac{\left(\xi^{2}-1\right)^{\frac{1}{2}}(\mathrm{~d} x / \mathrm{d} \xi)}{\xi-\zeta} \mathrm{d} \xi+\left(\zeta^{2}-1\right)^{-\frac{1}{2}} P(\zeta)
$$

where the function $\left(\zeta^{2}-1\right)^{\frac{1}{2}}$ is defined in the entire $\zeta$-plane with branch cuts from $-\infty$ to -1 and from 1 to $\infty$ along the $\xi$-axis so that $\left(\zeta^{2}-1\right)^{\frac{1}{2}} \rightarrow \zeta$ as $|\zeta| \rightarrow \infty$, for $0<\arg \zeta<\pi$ (note that the branch cut 
for the function $\left(\zeta^{2}-1\right)^{\frac{1}{2}}$ for this perturbation part is different from that for the basic problem as described after (83b)). The arbitrary function $P(\zeta)$ is real on the real $\zeta$-axis, and hence can be expanded into the Laurent's series

$$
P(\zeta)=\sum_{n=-\infty}^{\infty}\left[c_{n}\left(\zeta-\zeta_{\infty}\right)^{n}+\bar{c}_{n}\left(\zeta-\bar{\zeta}_{\infty}\right)^{n}\right]
$$

Since $z \sim\left(\zeta-\zeta_{\infty}\right)^{-1}$ as $|z| \rightarrow \infty$, it is necessary to have $c_{n}=0$ for $\mathrm{n} \leq-2$ in order that the perturbation velocity $\mathrm{w}_{\mathrm{l}}$ vanishes at infinity. Furthermore, we must impose $c_{n}=0$ for $n \geq 0$ if we require the pressure at $|\zeta|=\infty$ to be finite. Finally, application of (30) yields

$$
\begin{aligned}
& P(\zeta)=c_{-1}\left(\zeta-\zeta_{\infty}\right)^{-1}+\bar{c}_{-1}\left(\zeta-\bar{\zeta}_{\infty}\right)^{-1}, \\
& c_{-1}=-\frac{Q}{2 \pi}\left(\zeta_{\infty}^{2}-1\right)^{\frac{1}{2}}=-\frac{Q}{4 \pi}\left(W^{-1}-W\right),
\end{aligned}
$$

where use has been made of (84) and (85).

Transforming the variable $\zeta$ in the above solution into w by (83) we obtain

$$
G=\frac{2 b w^{2}}{\pi\left(1-w^{2}\right)} \int_{-1}^{1} g(w, u) d u-\frac{Q_{1} w^{2}}{\pi\left(1-w^{2}\right)}\left[\frac{1-W^{2}}{(w-W)(w W-1)}+\frac{1-\bar{W}^{2}}{(w-\bar{W})(w W-1)}\right]
$$

where

$$
g(w, u)=\frac{1}{u} \frac{d f_{0}}{d u} \frac{1-u^{2}}{(u-w)(u w-1)} .
$$

In particular, on the rigid plate, $-1<w<1, \quad R \ell G=\partial \varphi_{1} / \partial \xi$, and

$$
\frac{\partial \varphi}{\partial \xi}=\frac{2 w^{2}}{\pi\left(1-w^{2}\right)}\left\{b_{1}(P \cdot V .) \int_{-1}^{1} g(w, u) d u-\frac{Q^{2}}{2}\left[\frac{1-W^{2}}{(w-W)(w W-1)}+\frac{1-\bar{W}^{2}}{(w-\bar{W})(w \bar{W}-1)}\right]\right\}
$$


where (P.V.) denotes the Cauchy principal value of the integral.

The normal force $\mathrm{N}_{1}$ acting on the plate due to the acceleration is given by

$$
N_{1}=\rho \int_{-1}^{1} x(w) \frac{\partial \varphi}{\partial \xi} \frac{\partial \xi}{\partial w} d w
$$

where $\partial \varphi_{1} / \partial \xi$ is given by (95), and $\partial \xi / \partial w$ can be obtained from (83). As in the previous cases, we write

$$
C_{N_{1}}=-\left(\frac{l b}{U^{2}}\right) \Gamma_{b_{1}}-\left(\frac{Q_{1}}{U^{2}}\right) \Gamma_{Q_{1}},
$$

then, from (96),

$$
\begin{aligned}
& \Gamma_{b_{1}}=\frac{2}{\pi}(P \cdot V .) \int_{-1}^{1} x(w) d w \int_{-1}^{1} g(w, u) d u, \\
& \Gamma_{Q_{1}}=-\frac{1}{\pi} \int_{-1}^{1} x(w)\left[\frac{1-w^{2}}{(w-W)(w W-1)}+\frac{1-\bar{W}^{2}}{(w-\bar{W})(w \bar{W}-1)}\right] d w .
\end{aligned}
$$

These integrals have been computed numerically with an IBM 7090, the final result of $\Gamma_{\mathrm{b}}$ and $\Gamma_{Q}$ is shown in figures 8 and 9 for $\alpha=90^{\circ}, 75^{\circ}$ and $45^{\circ}$. The result of the special case $\alpha=\pi / 2$ is also compared with the other flow models in figures 6 and 7. For the special case $\alpha=\pi / 2$, we deduce from (98) the following expression for small $\sigma$

$$
\Gamma_{b}=0.8448\left[1+0.067 \sigma^{2}(1-\sigma)+\left(\sigma^{4}\right)\right]
$$

which is the special case already noted by $\mathrm{Wu}$ [6]. 


\section{Conclusion}

In the foregoing sections we have treated the accelerating motion of an inclined plate in Kirchhoff flow and in the finite-cavity flow, and that of a pure-drag plate in Riabouchinsky flow and in the re-entrant jet flow. From these results we make conclusions by pointing out the following features of interest.

(i) Effect of the cavitation number $\sigma$ For $0<\sigma<1$, the force coefficient $I_{b}$ due to the normal acceleration evaluated by using three different cavity-flow models is nearly identical, tending to 0.8448 , the Kirchhoff-Helmholtz limit, as $\sigma \longrightarrow 0$, and being very insensitive to $\sigma$ in this range. As $\sigma$ increases further from $1, \Gamma_{b}$ increases very slowly compared with the rate of increase of the steady state drag coefficient, which has the factor $(1+\sigma)$ approximately. For a fixed $\sigma$ in this region, the value of $\Gamma_{b}$ by the wake model is higher than that of the Riabouchinsky model, which is in turn higher than that of the reentrant jet model. This result indicates that in all practical ranges of $\sigma$, it is unimportant to consider the effect of $\sigma$ for the small-time limit or the large frequency limit.

(ii) Effect of incidence $\alpha$ - For moderate and large $\sigma$, the force coefficient $\Gamma_{b_{1}}$ increases faster with increasing $\sigma$, the smaller is the value $\alpha$. This result should be expected on the physical standpoint since there contains more liquid in the circular cylinder spanned by the plate at smaller $\alpha$. This effect of $\alpha$, however, becomes insignificant for $\sigma$ less than order unity.

(iii) Dffect of change in cavity volume The force coefficient $\Gamma_{\mathrm{Q}}$ due to the change in cavity volume displays wider differences between diferent flow-models. All these models show, however, that $\Gamma_{Q} \rightarrow 0$ rather rapidly as $\sigma \rightarrow 0$, saying that the effect on the drag force of removing fluid at infinity is insignificant when the cavity is sufficiently long. This analysis also justifies the assumption that $p$ is bounded at infinity introduced in some of the previous works $[4,6,7,8,9,18]$ 
in the case of unsteady flows with infinite cavities. As shown by this analysis, this assumption actually turns out to be a necessary condition for the existence of the solution in the case of $\sigma=0$.

This work is sponsored in part by the Office of Naval Research of the U. S. Navy, under contract Nonr 220(35) at the California Institute of Technology. Reproduction in whole or in part is permitted for any purpose of the United States Government. The assistances rendered by Mr. Barry Bass and Mrs. Zora Harrison in the numerical computation, by Miss Cecilia Lin in the graphic work and Mrs. Barbara Hawk in the preparation of the manuscript are greatly appreciated by the authors. 


\section{References}

[1] Gilbarg, D.: Jets and Cavities; Encylopedia of Physics, Vol. IX Springer-Verlag, Berlin, (1960).

[2] Birkhoff, G. and Zarantonello, E. H.: Jets, Wakes and Cavities. Academic Press, New York, (1957).

[3] Wang, D. P.: A perturbation theory for unsteady cavity flows, Ph.D. Thesis, California Institute of Technology, Pasadena, (1962).

[4] Gurevich, M.E.: The impact on a lamina with discontinuous streamline flow, Prikl. Math. Mekh. 16, 116-118,(1952). Translated into English by Gerta and Hirsh Cohen, RPI Math. Trans. No. 3, (1959), Rensselaer Polytechn. Inst., New York.

[5] Wu, T. Y.: A free streamline theory for two-dimensional fully cavitated hydrofoils, J. Math. Phys. 35, 236-265,(1956).

[6] Wu, T. Y .: Unsteady supercavitating flows. Second Symposium on Naval Hydrodynamics, Washington D.C., 293-313,(1958).

[7] Timman, R.: A general linearized theory for cavitating hydrofoils in nonsteady flow. Second Symposium on Naval Hydrodynamics, Washington D.C., 559-579,(1958).

[8] Geurst, J. A.: Some investigations of a linearized theory for unsteady cavity flows. Arch. Rat. Mech. Anal. 5, 315-346, (1960).

[9] Parkin, B.R.: Fully cavitating hydrofoils in nonsteady motion. Calif. Inst. of Tech. Rept. No. Eng. 85-2, Pasadena, (1957).

[10] Muskhelishvili, N. I.: Singular Integral Equations. P. Noordhoff Ltd., Groningen, Holland, 235 - 238, (1946).

[11] Lamb, H.: Hydrodynamics. Dover Publications, Sixth Ed. $102-103,(1945)$. 
[12] Riabouchinsky, D.: On steady fluid motion with free surface. Proc. Lond. Math. Soc. (2), 19, 206-215,(1920).

[13] Joukowsky, N.E.: "I. A modification of Kirchhoff's method of determining a two dimensional motion of a fluid given a constant velocity along an unknown streamline. II. Determination of the motion of a fluid for any condition given on a streamline." Works of N. E. Joukowsky, Vol. II, Is sue 3, Trans. CAHI, No. 41, (1930).

[14] Roshko, A.: "A new hodograph for free-streamline theoryo" NACA Tech. Note $3168,(1954)$.

[15] Eppler, R.: Journal of Rat. Mech. and Analysis. Vol. 3, $591-644,(1954)$.

[16] Wu, T. Y.: Journal of Fluid Mech. Vol. 13, Part 2, 161 - 181, (1962).

[17] Byrd, P. F. and Friedman, M. D.: Handbook of Elliptic Integrals. Springer-Verlag, Berlin (1954).

[18] Woods, L. C.: Proc. Roy. Soc. London, A, 229, 152-180, (1955); $229,235-250,(1955) ; 239,328-337,(1957)$. 

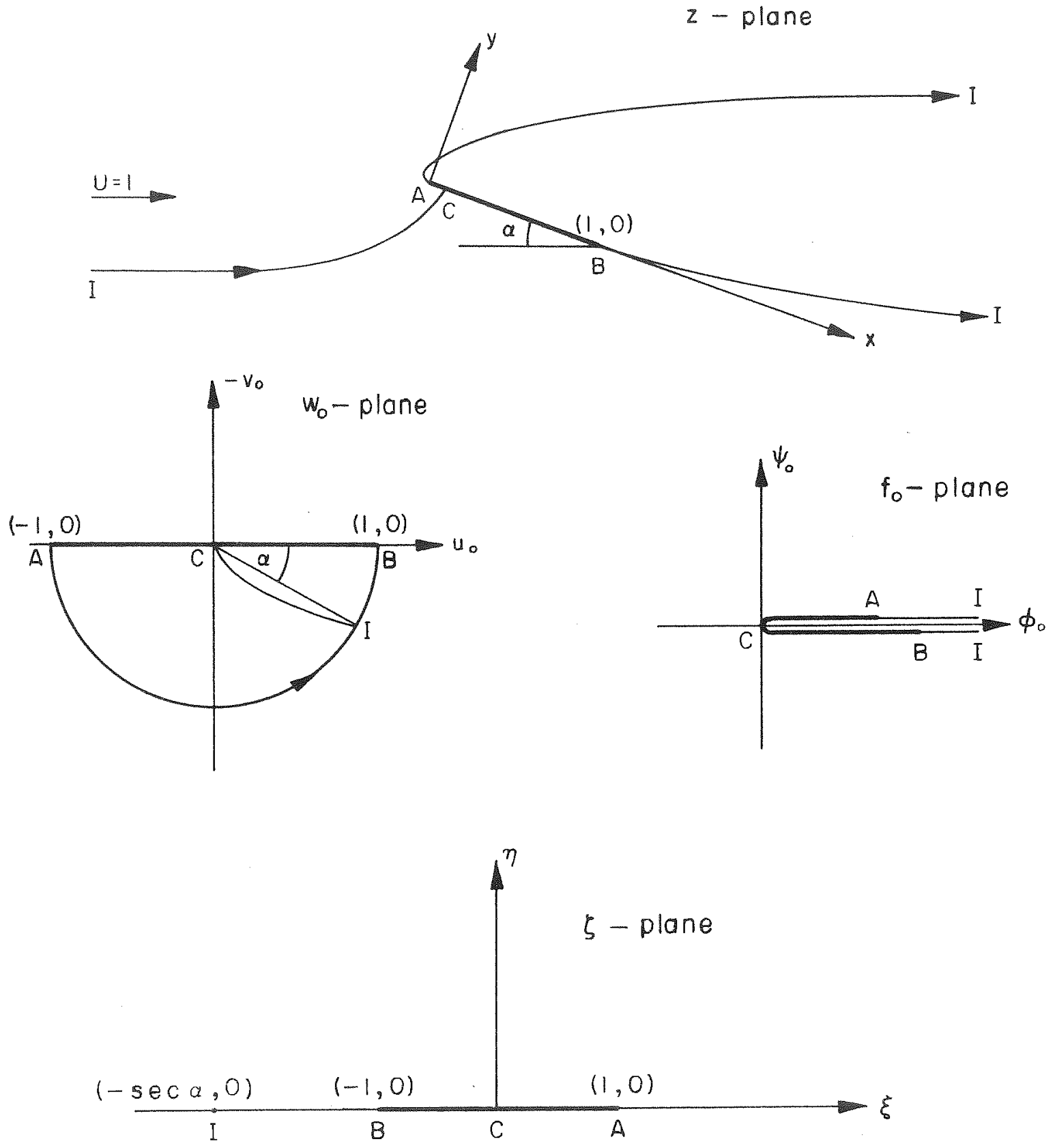

Figure 1. Inclined lamina in Kirchhoff flow and its conformal mapping planes. 

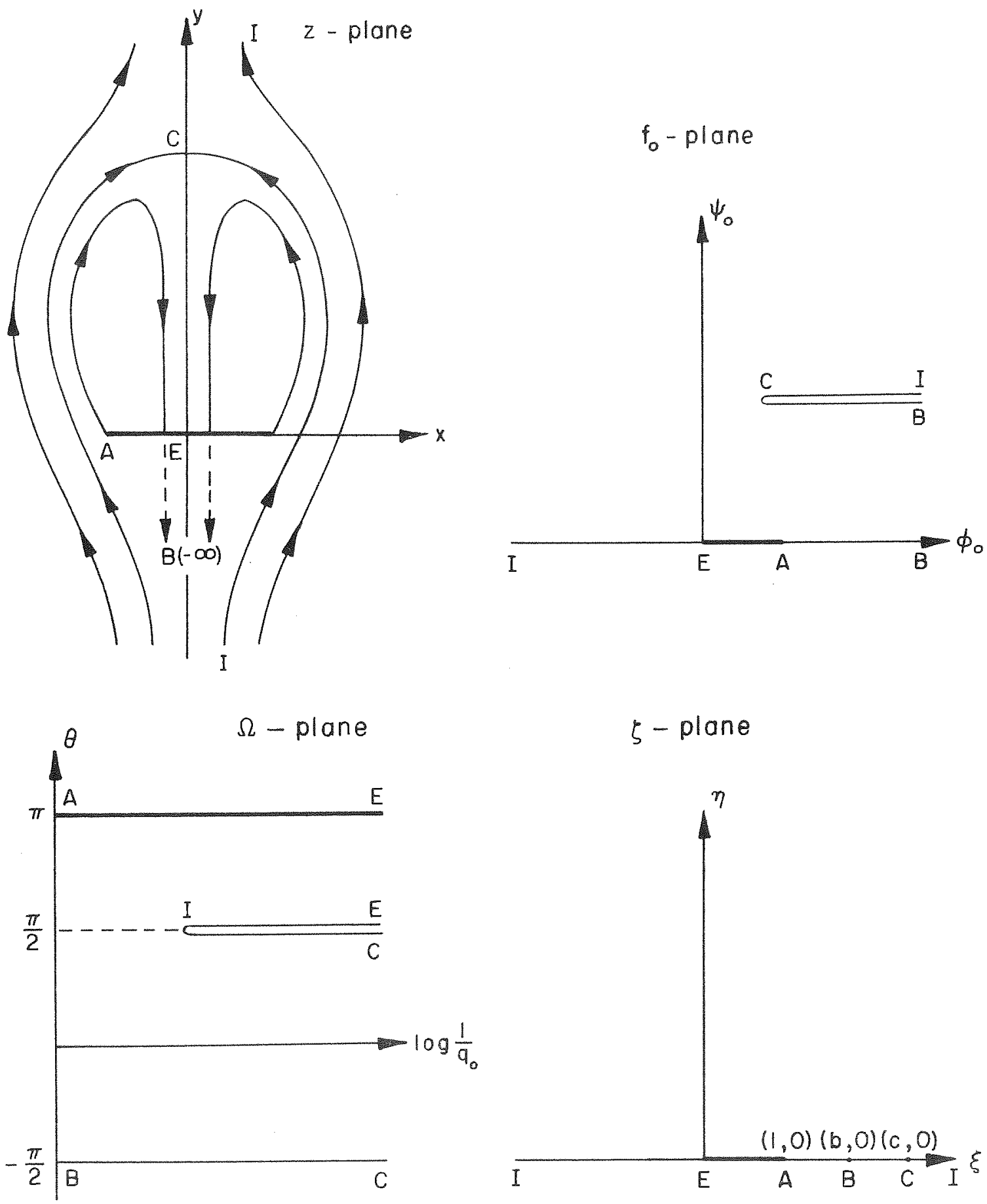

$\zeta$ - plane

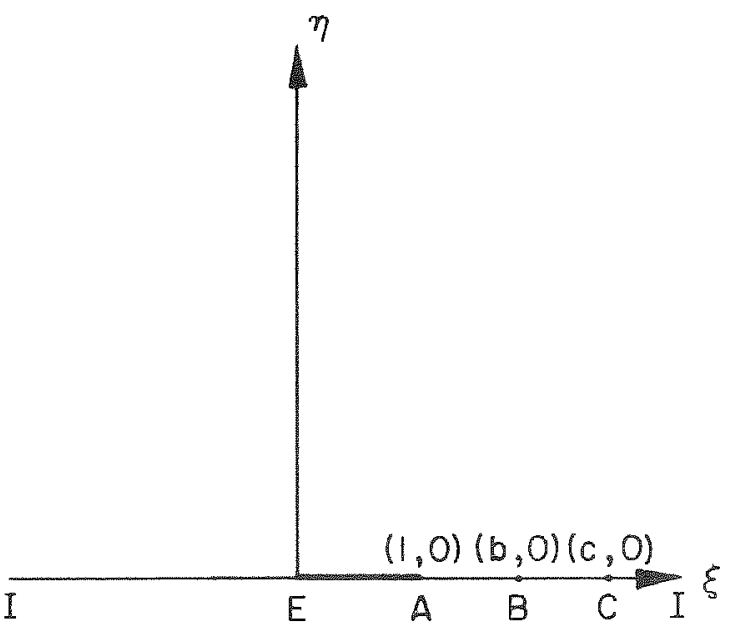

Figure 2. Re-entrant jet flow past a flat plate and its conformal mapping planes. 

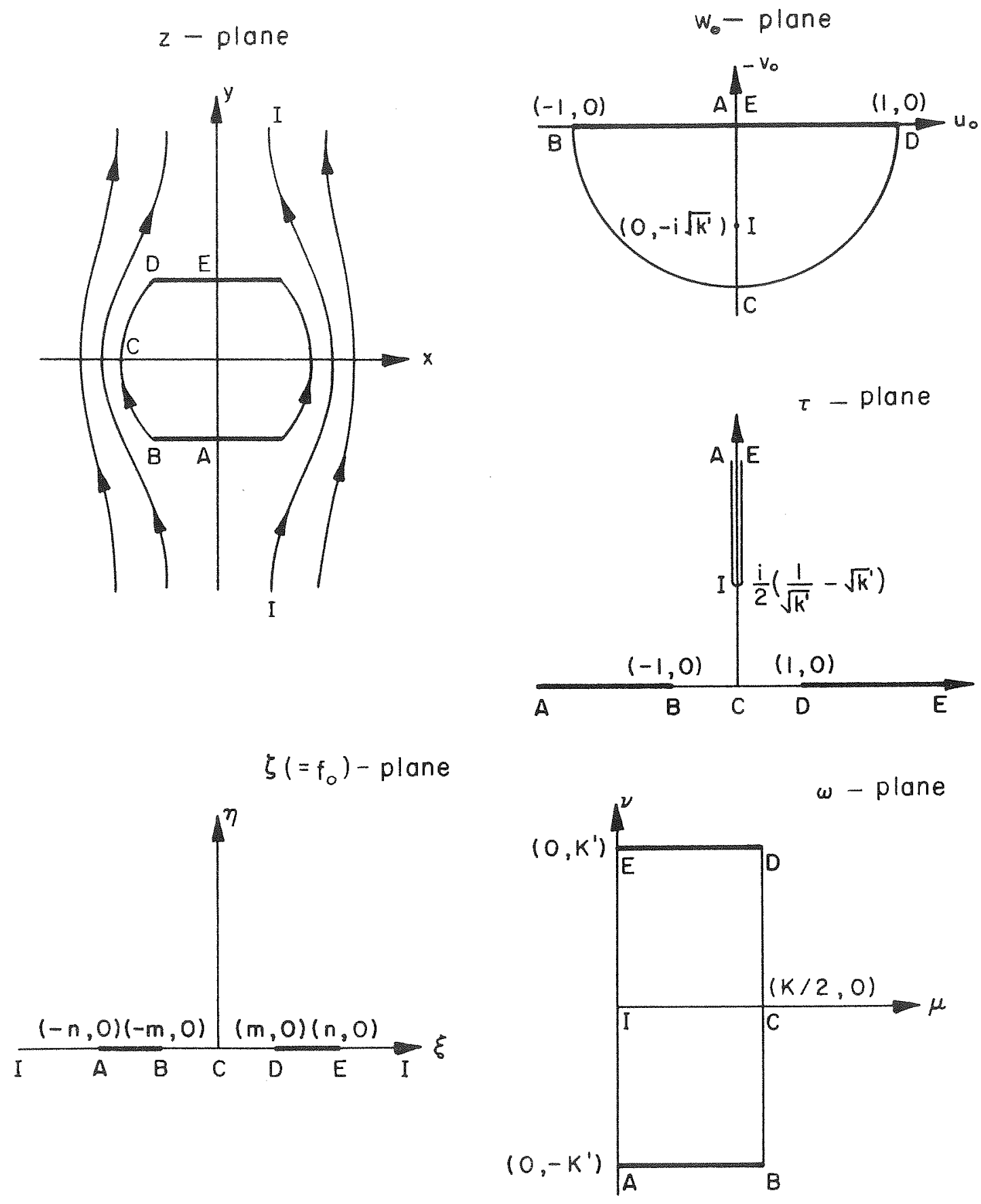

Figure 3. Riabouchinsky's model for a flat plate and its conformal mapping planes. 

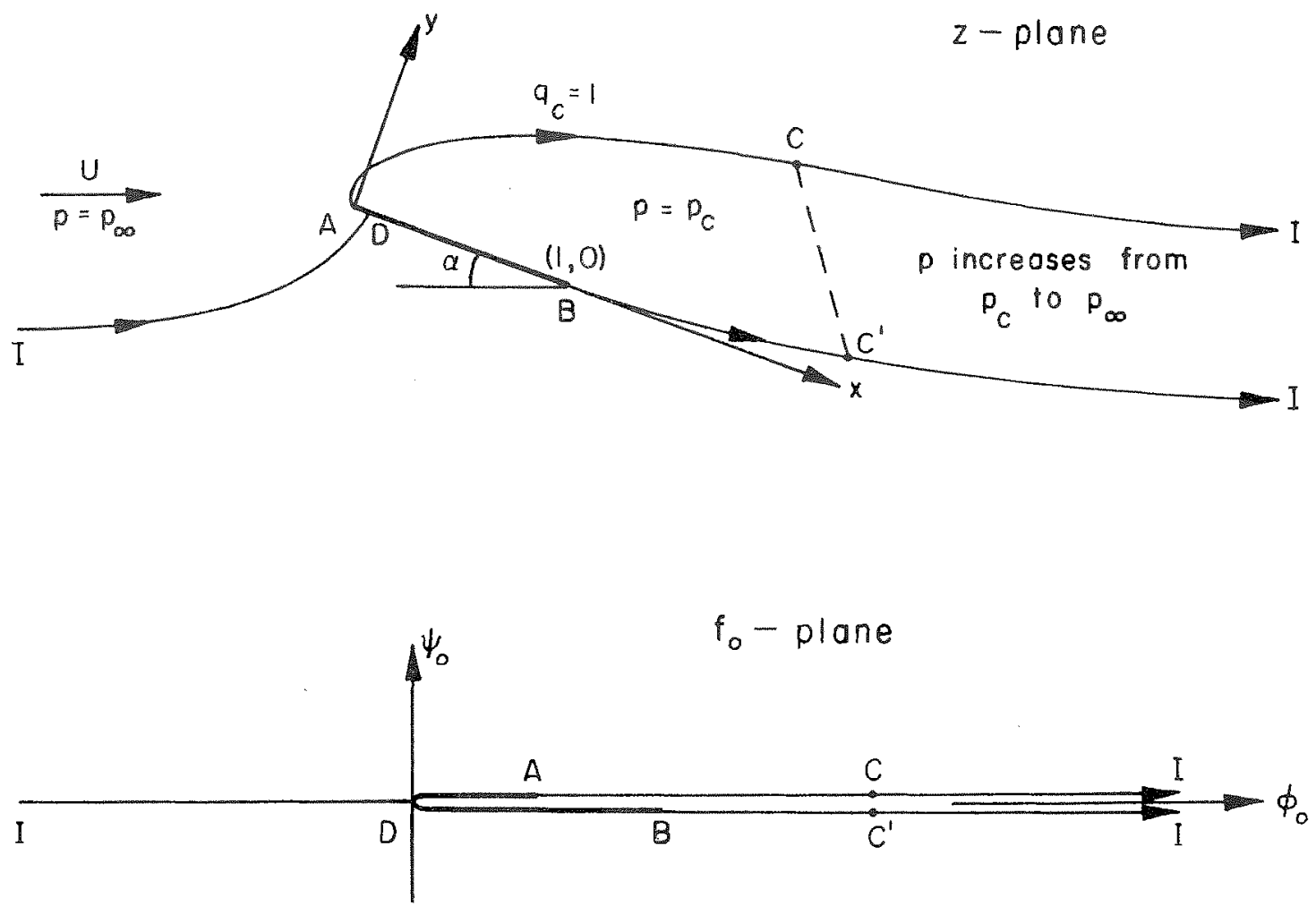

$w_{0}-$ plane

$\zeta$-plane
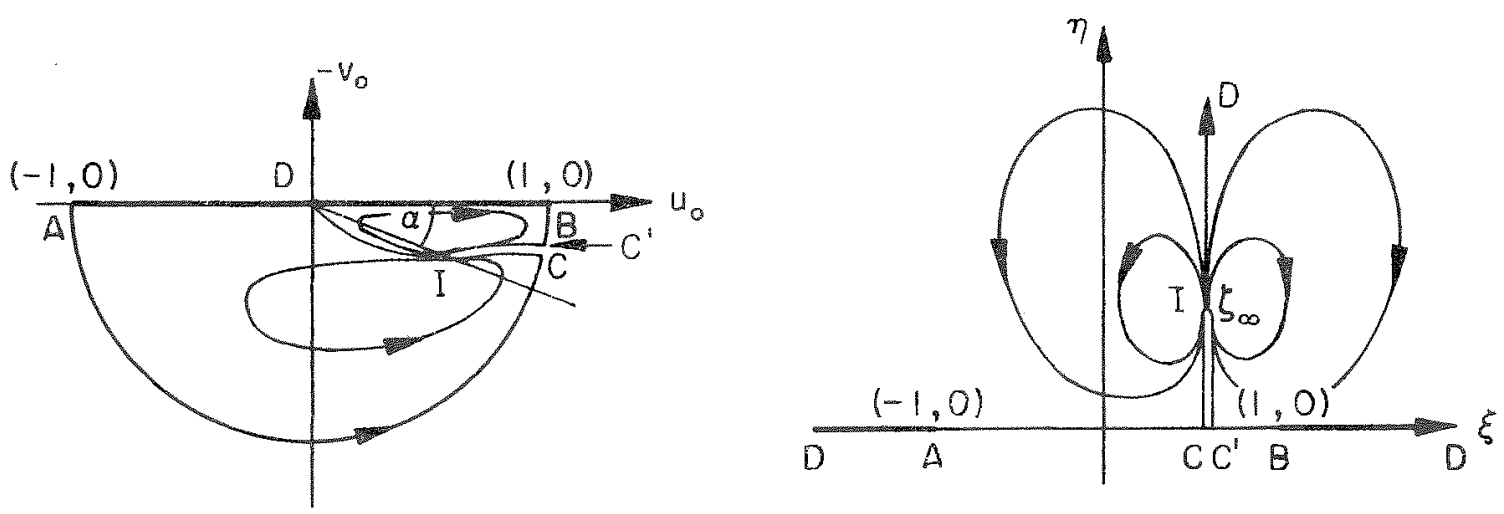

Figure 4. A wake model for an oblique plate with a finite cavity and its conformal mapping planes. 


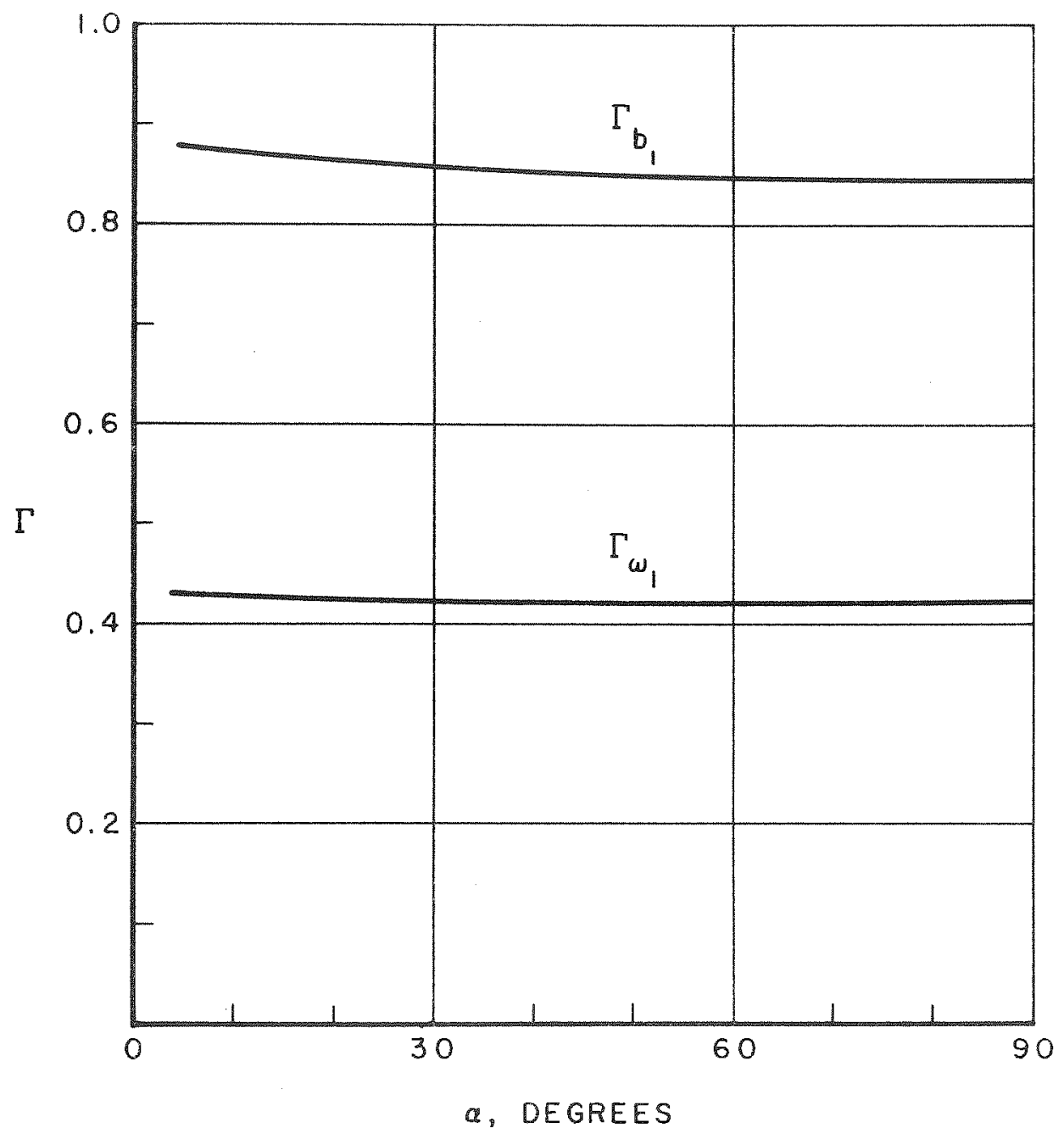

Figure 5. Normal force coefficients for inclined lamina in Kirchhoff flow. 


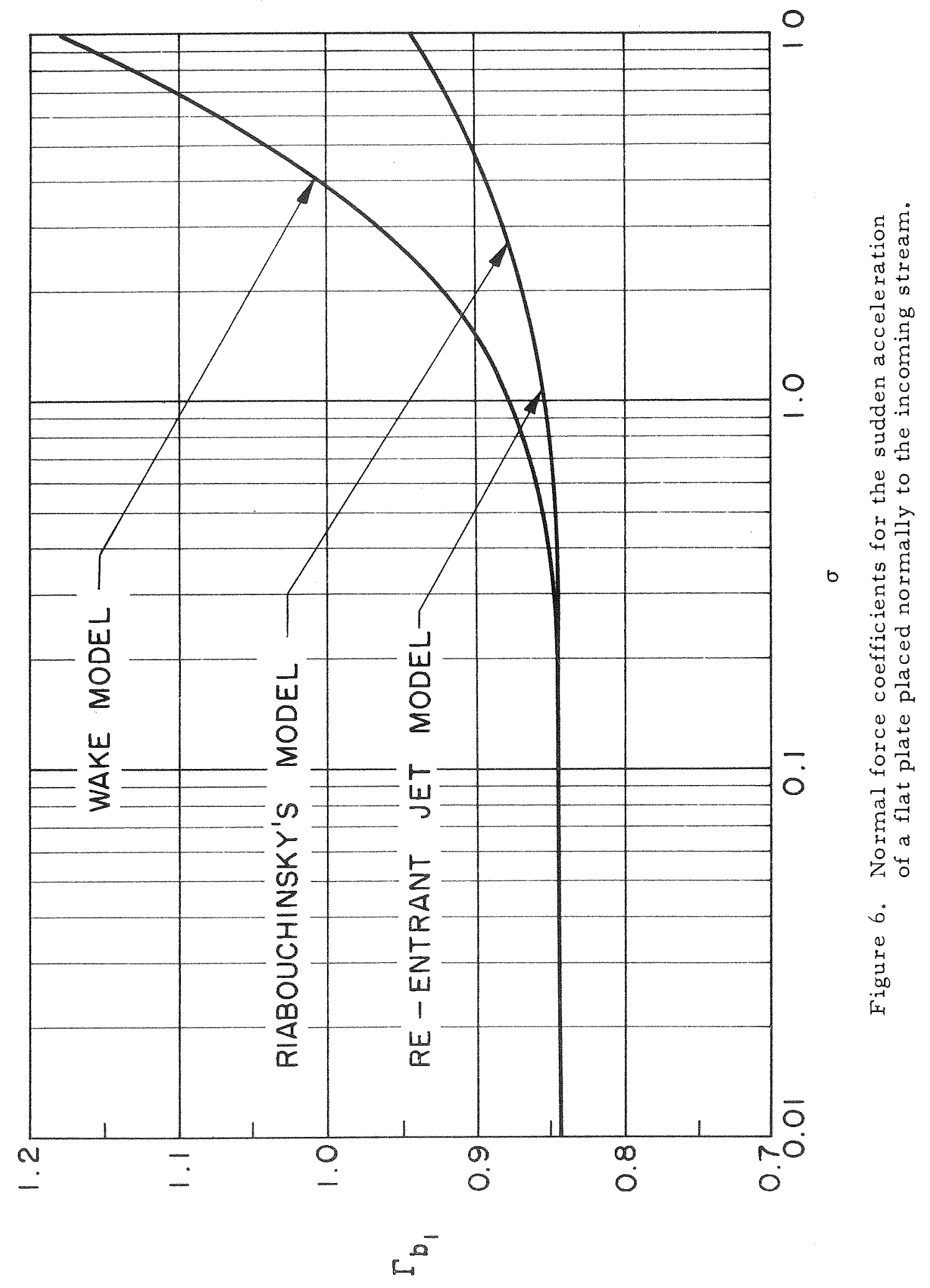




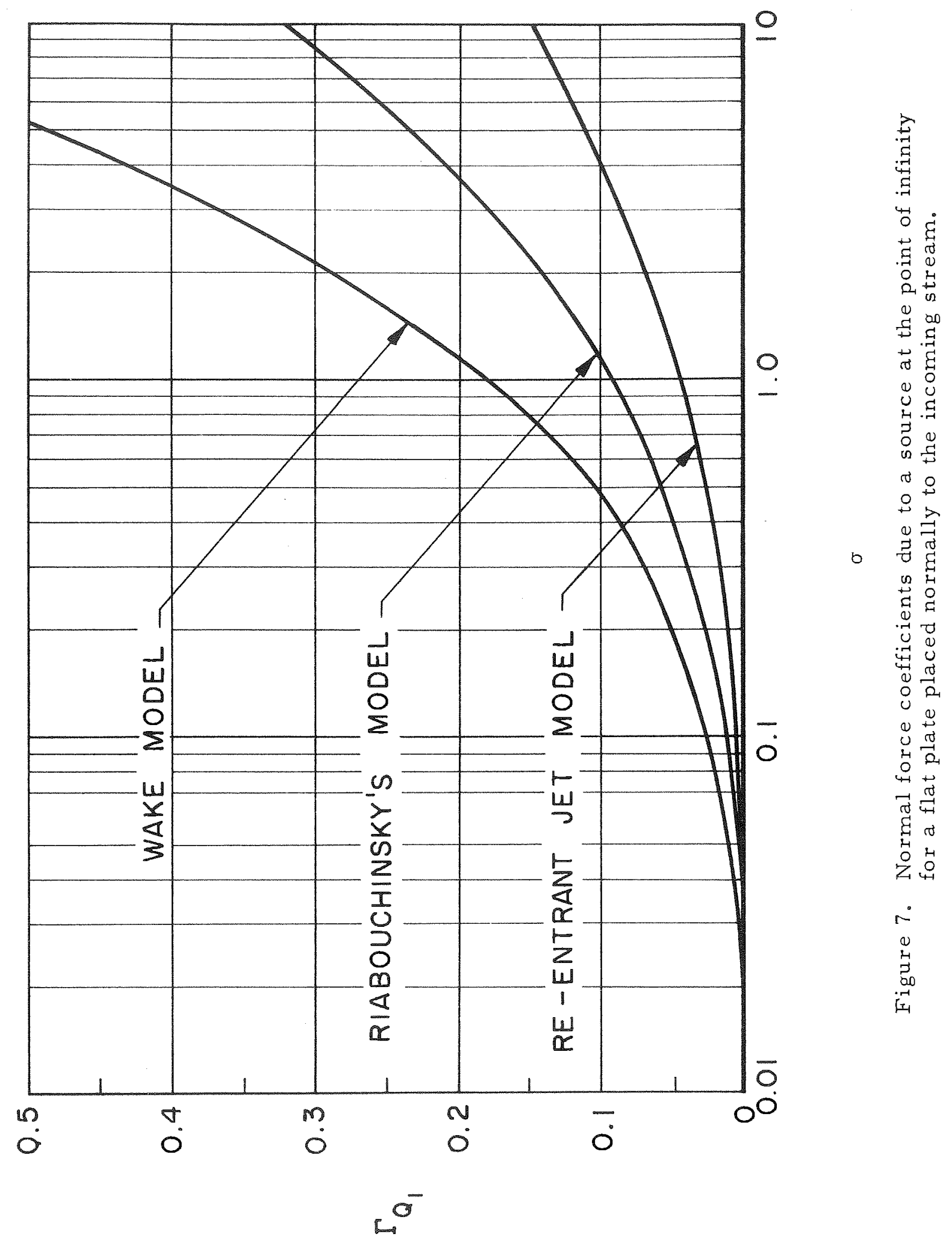




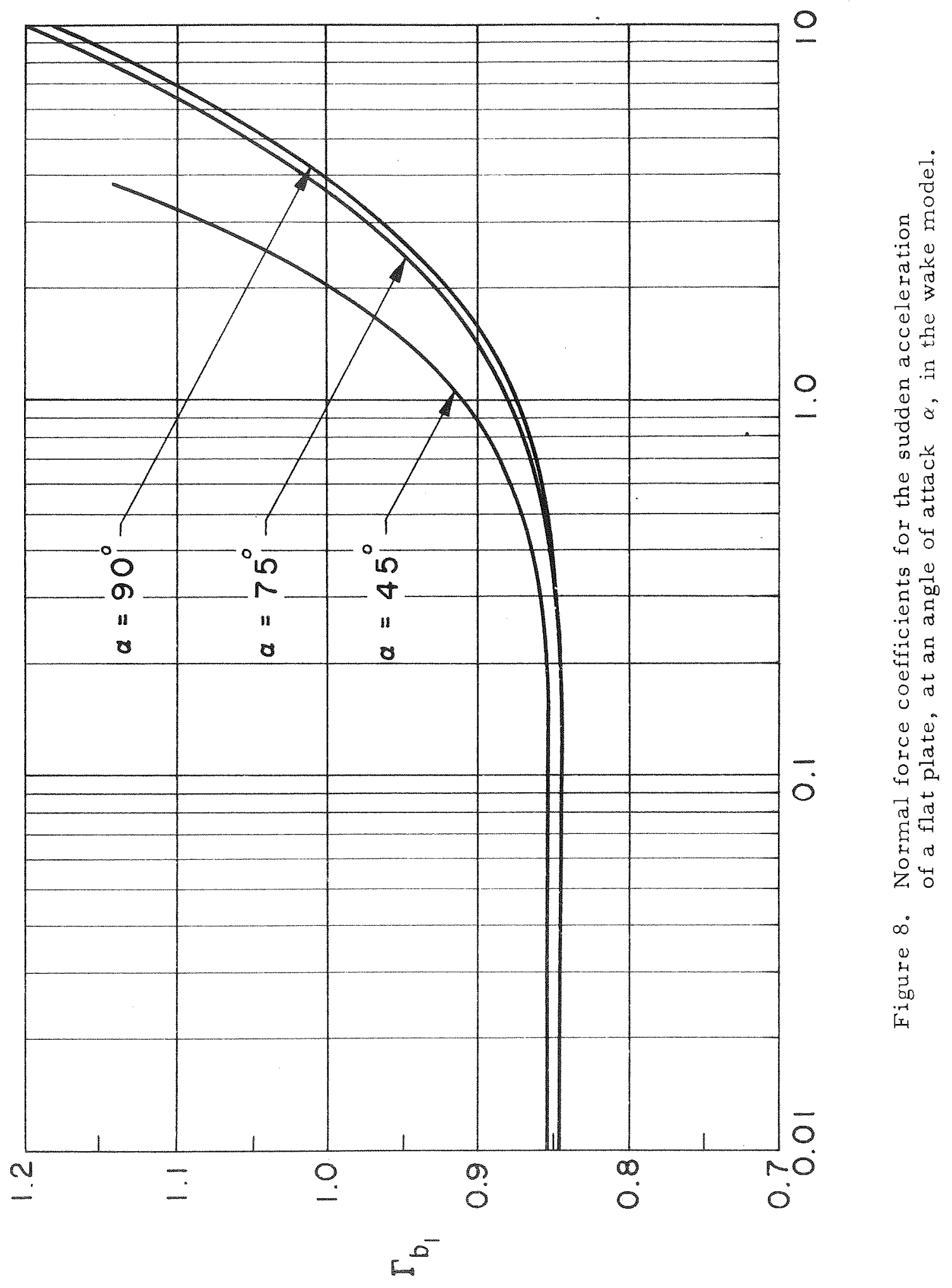




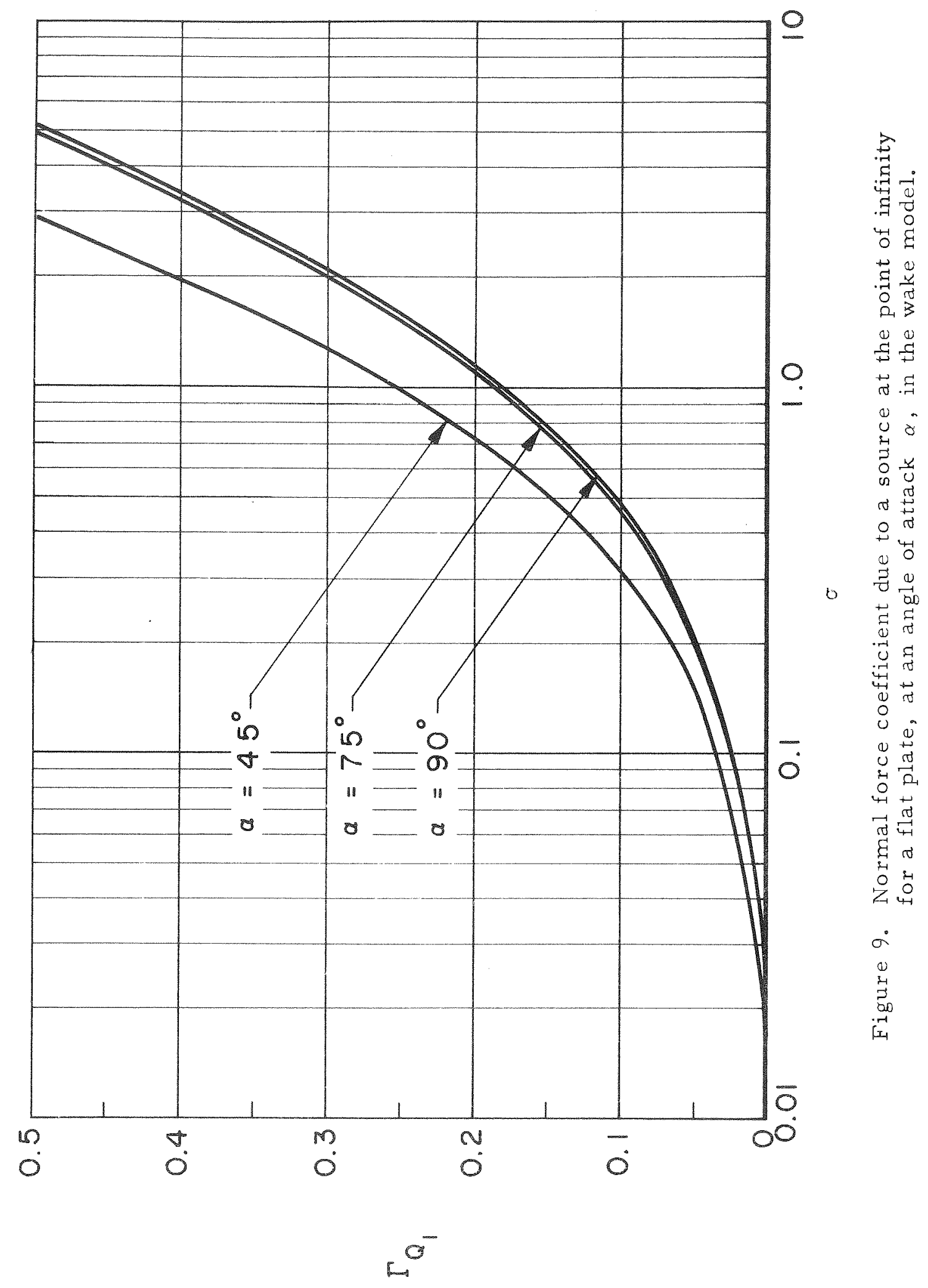




\section{DISTRIBUTION LIST FOR UNCLASSIFIED TECHNICAL REPORTS \\ ISSUED UNDER}

CONTRACT Nonx 220(35), TASK NR 062-230

(Single copies unless otherwise specified)

Chief of Naval Research Department of the Navy Washington 25, D.C.

Attn: Codes 438 (3)

461

463

466

Commanding Officer

Office of Naval Research

Branch Office

495 Summer Street

Boston 10, Massachusetts

Commanding Officer

Office of Naval Research

Branch Office

207 West 24th Street

New York ll, New York

Commanding Officer

Office of Naval Research

Branch Office

1030 East Green Street

Pasadena, California

Commanding Officer

Office of Naval Research

Branch Office

1000 Geary Street

San Francisco 9, California

Commanding Officer

Office of Naval Research

Branch Office

Box 39, Navy No. 100

Fleet Post Office

New York, New York

Director

Naval Research Laboratory

Washington 25, D. C.

Attn: Code 2027 (6)

Chief, Bureau of Naval Weapons

Department of the Navy

Washington 25, D. C.

Attn: Codes RUAW -r

RRRE

RAAD

RAAD - 222

DIS -42

Commander

U. S. Naval Ordnance Test Station

China Lake, California

Attn: Code 753

$\begin{array}{lc}\text { Chief, Bureau of Ships } \\ \text { Department of the Navy } \\ \text { Washington 25, D. C. } \\ \text { Attn: Codes } & 310 \\ & 312 \\ & 335 \\ & 420 \\ & 421 \\ & 440 \\ & 442 \\ & 449\end{array}$

Chief, Bureau of Yards and Docks Department of the Navy Washington 25, D. C.

Attn: Code D-400

Commanding Officer and Director David Taylor Model Basin

Washington 7, D. C.

Attn: Codes 108

142

500

513

520

525

526

$526 \mathrm{~A}$

530

533

580

585

589

591

$591 \mathrm{~A}$

700

Commander

U.S. Naval Ordnance Test Station

Pasadena Annex

3202 E. Foothill Blvd.

Pasadena 8, California

Attn: Code P-508

Commander

Planning Department

Portsmouth Naval Shipyard

Portsmouth, New Hampshire

Commander

Planning Department

Boston Naval Shipyard

Boston 29, Massachusetts 
Commander

Planning Department

Pearl Harbor Naval Shipyard

Navy No. 128, Fleet Post Office

San Francisco, California

Commander

Planning Department

San Francisco Naval Shipyard

San Francisco 24, California

Commander

Planning Department

Mare Island Naval Shipyard

Vallejo, California

Commander

Planning Department

New York Naval Shipyard

Brooklyn 1, New York

Commander

Planning Department

Puget Sound Naval Shipyard

Bremerton, Washington

Commande $r$

Planning Department

Philadelphia Naval Shipyard

U. S. Naval Base

Philadelphia 12, Pennsylvania

Commander

Planning Department

Norfolk Naval Shipyard

Portsmouth, Virginia

Commander

Planning Department

Charleston Naval Shipyard

U. S. Naval Base

Charleston, South Carolina

Commander

Planning Department

Long Beach Naval Shipyard

Long Beach 2, California

Commander

Planning Department

U. S. Naval Weapons Laboratory

Dahlgren, Virginia

Commander

U. S. Naval Ordnance Laboratory

White Oak, Maryland

Dr. A. V. Hershey

Computation and Exterior

Ballistics Laboratory

U. S. Naval Weapons Laboratory

Dahlgren, Virginia
Superintendent

U.S. Naval Academy

Annapolis, Maryland

Attn: Library

Superintendent

U. S. Naval Postgraduate School

Monterey, California

Commandant

U. S. Coast Guard

1300 E. Street, N. W.

Washington, D. C.

Secretary Ship Structure Committee U. S. Coast Guard Headquarters

1300 E Street, N.W.

Washington, D.C.

Commander

Military Sea Transportation Service

Department of the Navy

Washington 25, D. C.

U. S. Maritime Administration

GAO Building

441 G Street, N. W.

Washington, D. C.

Attn: Division of Ship Design

Division of Research

Superintendent

U. S. Merchant Marine Academy

Kings Point, Long Island, New York

Attn: Capt. L. S. McCready

(Dept. of Engineering)

Commanding Officer and Director

U. S. Navy Mine Defense Laboratory

Panarna City, Florida

Commanding Officer

NROTC and Naval Administrative

Massachusetts Institute of Technology

Cambridge 39, Massachusetts

U. S. Army Transportation Research and

Development Command

Fort Eustis, Virginia

Attn: Marine Transport Division

Mr.J.B. Parkinson

National Aeronautics and Space

Administration

1512 H Street, N.W.

Washington 25, D. C.

Director

Langley Research Center

Langley Station

Hampton, Virginia

Attn: Mr. I. E. Garrick

Mr. D. J. Marten 
Director Engineering Sciences Division National Science Foundation 1951 Constitution Avenue, N. W. Washington $25, \mathrm{D}$. C.

Director

National Bureau of Standards

Washington 25, D. C.

Attn: Fluid Mechanics Division (Dr.G. B. Schubauer)

Dr. G. H, Keulegan

Dr.J.M. Franklin

Armed Services Technical Information Agency

Arlington Hall Station

Arlington 12, Virginia (10)

Office of Technical Services

Department of Commerce

Washington 25, D. C.

California Institute of Technology

Pasadena 4, California

Attn: Professor M. S. Plesset

Professor T. Y. Wu

Professor A. J.Acosta

University of California

Department of Engineering

Los Angeles 24, California

Attn: Dr. A. Powell

Director

Scripps Institute of Oceanography

University of California

La Jolla, California

Professor M. L. Albertson

Department of Civil Engineering

Colorado $A$ and $M$ College

Fort Collins, Colorado

Professor J.E. Cermak

Department of Civil Engineering

Colorado State University

Fort Collins, Colorado

Professor W. R. Sears

Graduate School of Aeronautical Engineering Ordnance Research Laboratory

Cornell University

Ithaca, New York

State University of Iowa

Iowa Institute of Hydraulic Research

Iowa City, Iowa

Attn: Dr. H. Rouse

Dr. L. Landweber

Massachusetts Institute of Technology

Cambridge 39, Massachusetts

Attn: Department of Naval Architecture Woods Hole, Massachusetts

and Marine Engineering

Professor A. T. Ippen
Harvard University

Cambridge 38, Massachusetts

Attn: Professor G. Birkhoff

(Dept. of Mathematics)

Professor G. F. Carrier

(Dept. of Mathematics)

University of Michigan

Ann Arbor, Michigan

Attn: Professor R. B. Couch (Dept. of Naval Architecture)

Professor W. W. Willmarth

(Aero. Engineering Department)

Professor M. S. Uberoi

(Aero. Engineering Department)

Dr. L. G. Straub, Director

St. Anthony Falls Hydraulic Laboratory

University of Minnesota

Minneapolis 14, Minnesota

Attn: Mr.J.N. Wetzel

Professor B. Silberman

Professor J. J. Foody

Engineering Department

New York State University Maritime College

Fort Schulyer, New York

New York University

Institute of Mathematical Sciences

25 Waverly Place

New York 3, New York

Attn: Professor J. Keller

Professor J. J. Stoker

The Johns Hopkins University

Department of Mechanical Engineering

Baltimore 18, Maryland

Attn: Professor S. Corrsin

Massachusetts Institute of Technology

Department of Naval Architecture and

Marine Engineering

Cambridge 39, Massachusetts

Attn: Professor M. A. Abkowitz, Head

Dr. G. F. Wislicenus

Pennsylvania State University

University Park, Pennsylvania

Attn: Dr. M. Sevik

Professor R. C. DiPrima

Department of Mathematics

Rensselaer Polytechnic Institute

Troy, New York

Director

Woods Hole Oceanographic Institute

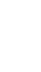

Professor O. M. Phillips 
Stevens Institute of Technology

Davidson Laboratory

Castle Point Station

Hoboken, New Jersey

Attn: Mr。D. Savitsky

Mr.J.P. Breslin

Mr. C. J. Henry

Mr.S. Tsakonas

Webb Institute of Naval Architecture

Crescent Beach Road

Glen Cove, New York

Attn: Professor E. V. Lewis

Technical Library

Executive Director

Air Force Office of Scientific Research

Washington $25, \mathrm{D}$. C.

Attn: Mechanics Branch

Commander

Wright Air Development Division

Aircraft Laboratory

Wright-Pattern Air Force Base, Ohio

Attn: Mr.W. Mykytow, Dynamics

Branch

Cornell Aeronautical Laboratory

4455 Genesee Street

Buffalo, New York

Attn: Mr. W. Targoff

$\mathrm{Mr}$. R. White

Massachusetts Institute of Technology

Fluid Dynamics Research Laboratory

Cambridge 39, Massachusetts

Attn: Professor H. Ashley

Professor M. Landahl

Professor J. Dugundji
Shipsmodelltanken

Trondheim, Norway

Attn: Professor J. K. Lunde

Versuchsanstalt fur Wasserbau and Schiffbau

Schleuseninsel im Tiergarten

Berlin, Germany

Attn: Dr. S. Schuster, Director

Dr. H. Schwanecke

Dr. Grosse

Technische Hogeschool

Institut voor Toegepaste Wiskunde

Julianalaan 132

Delft, Netherlands

Attn: Professor R. Timman

Bureau D'Analyse et de Recherche Appliquees

47 Avenue Victor Bresson

Issy-Les-Moulineaux

Seine, France

Attn: Professor Siestrunck

Netherlands Ship Model Basin

Wageningen, The Netherlands

Attn: Dr. Ir. J. D. vanManen

National Physical Laboratory

Teddington, Middlesex, England

Attn: Mr. A. Silverleaf, Supe rintendent Ship Division

Head, Aerodynamics Division

Head, Aerodynamics Department

Royal Aircraft Establishment

Farnborough, Hants, England

Attn: Mr. M. O. W. Wolfe

Dr. S. F. Hoerner

148 Busteed Drive

Hamburgische Schiffbau-Versuchsanstalt Bramfelder Strasse 164

Hamburg 33, Germany

Attn: Dr. O. Grim

Dr. H.W. Lerbs

Institut fur Schiffbau der

Universitat Hamburg

Berliner Tor 21

Hamburg 1, Germany

Attn: Prof. G. P. Weinblum, Director
Midland Park, New Jersey

Boeing Airplane Company

Seattle Division

Seattle, Washington

Attn: Mr. M. J. Turner

Electric Boat Division

General Dynamics Corporation

Groton, Connecticut

Transportation Technical Research Institute Attn: Mr. Robert McCandliss 1-1057, Mejiro-Cho, Toshima-Ku

Tokyo, Japan

General Applied Sciences Labs., Inc.

Merrick and Stewart Avenues

Max-Planck Institut fur Stromungsforschun

Bottingerstrasse $6 / 8$

Wh estbury, Long Island, New York

Gottingen, Germany

Gibbs and Cox, Inc.

21 West Street

Attn: Dr. H. Reichardt

New York, New York

Hydro-og Aerodynamisk Laboratorium

Lyngby, Denmark

Attn: Professor Carl Prohaska

Lockheed Aircraft Corporation

Missiles and Space Division

Palo Alto, California

Attn: R. W. Kermeen 
Grumman Aircraft Engineering Corp. Bethpage, Long Island, New York

Attn: Mr. E. Baird

Mr.E. Bower

Mr.W.P.Carl

Midwest Research Institute 425 Volker Blvd.

Kansas City 10, Missouri

Attn: Mr. Zeydel

Director, Department of Mechanical Sciences

Southwest Research Institute

8500 Culebra Road

San Antonio 6, Texas

Attn: Dr. H, N. Abramson

Mr. G. Ransleben

Editor, Applied Mechanics

Review

Convair

A Division of General Dynamics

San Diego, California

Attn: Mr. R. H. Oversmith

Mr. H. T. Brooke

Hughes Tool Company

Aircraft Division

Culver City, California

Attn: Mr. M. S. Harned

Hydronautics, Incorporated

Pindell School Road

Howard County

Laurel, Maryland

Attn: Mr. Phillip Eisenberg

Rand Development Corporation

13600 Deise Avenue

Cleveland 10, Ohio

Attn: Dr. A. S. Iberall

U. S. Rubber Company

Research and Development Department

Wayne, New Jersey

Attn: Mr. L. M. White

Technical Research Group, Inc.

2 Aerial Way

Syosset, Long Island, New York

Attn: Mr. Jack Kotik

Mr。C. Wigley

Flat 102

6-9 Charterhouse Square

London, E. C. 1, England

AVCO Corporation

Lycoming Division

1701 K Street, N. W.

Apt. No. 904

Washington, D. C.

Attn: Mr. T. A. Duncan
Mr. J. G. Baker

Baker Manufacturing Company

Evansville, Wisconsin

Curtiss-Wright Corporation Research

Division

Turbomachinery Division

Quehanna, Pennsylvania

Attn: Mr. George H. Pedersen

Dr. Blaine R. Parkin

AiResearch Manufacturing Corporation

9851-9951 Sepulveda Boulevard

Los Angeles 45, California

The Boeing Company

Aero-Space Division

Seattle 24, Washington

Attn: Mr. R. E. Bateman

(Internal Mail Station 46-74)

Lockheed Aircraft Corporation

California Division

Hydrodynamics Research

Burbank, California

Attn: Mr. Bill East

National Research Council

Montreal Road

Ottawa 2, Canada

Attn: Mr. E. S. Turner

The Rand Corporation

1700 Main Street

Santa Monica, California

Attn: Technical Library

Stanford University

Department of Civil Engineering

Stanford, California

Attn: Dr. Byrne Perry

Dr. E. Y.Hsu

Dr. Hirsh Cohen

IBM Research Center

P. O. Box 218

Yorktown Heights, New York

Mr. David Wellinger

Hydrofoil Projects

Radio Corporation of America

Burlington, Massachusetts

Food Machinery Corporation

P. O. Box 367

San Jose, California

Attn: Mr. G. Tedrew

Dr. T. R. Goodman

Oceanics, Inc.

Technical Industrial Park

Plainview, Long Island, New York 
Professor Brunelle

Department of Aeronautical Engineering

Princeton University

Princeton, New Jersey

Commanding Officer

Office of Naval Research Branch Office

86 East Randolph Street

Chicago 1, Illinois 